\title{
Characterization of ground and excited electronic state deprotonation energies of systems containing double bonds using natural bond orbital analysis
}

\author{
Jay K. Badenhoop and Steve Scheiner \\ Department of Chemistry, Southern Illinois University, Carbondale, Illinois 62901
}

(Received 28 December 1996; accepted 14 June 1996)

\begin{abstract}
Natural bond orbital analysis is applied to the ground and excited states of a set of neutral, cationic, and anionic doubly bonded species $\mathrm{H}_{n} \mathrm{C}=\mathrm{XH}_{n}(\mathrm{X}=\mathrm{C}, \mathrm{N}, \mathrm{O})$ isoelectronic with ethylene. The character of the excitation is correlated with calculated charge shifts and geometry changes upon relaxation. For these planar molecules, depopulation of the $\pi$ bond or population of the $\pi^{*}$ antibond causes an out-of-plane twist or pyramidalization upon relaxation correlated to the amount of charge shift. These nonplanar distortions generally lower the energy more than changes in bond lengths and angles. Population of a $\sigma_{\mathrm{XH}}^{*}$ antibond by more than $\sim 0.4 e$ often leads to dissociation of that proton. The character and symmetry of the transition are used to match excited states in the protonated and deprotonated species so as to extract an excited state deprotonation energy. The vertical deprotonation energy of the $\pi \rightarrow \pi^{*}$ state tends to be higher than that of the ground state due to greater electronic destabilization of the deprotonated species, while Rydberg excited states take less energy to deprotonate. Adiabatic deprotonation energies can be greater or less than that of the ground state depending on whether the protonated or deprotonated species is more stabilized by geometry relaxation. (C) 1996 American Institute of Physics. [S0021-9606(96)01835-1]
\end{abstract}

\section{INTRODUCTION}

The topic of proton transfer has received much attention because of its importance in many chemical and biological processes, including solvation, ${ }^{1}$ enzyme catalysis, ${ }^{2}$ and ion transport. ${ }^{3}$ The phenomenon of photoinduced proton transfer has been studied experimentally ${ }^{4}$ and, with the development of $a b$ initio methods for the calculation of excited state wave functions $^{5,6}$ and including correlation, ${ }^{7}$ a variety of intermolecular and intramolecular proton transfer reactions have been studied theoretically. ${ }^{8}$ Electronic excitation alters the properties of the system so as to enable rapid transfer.

Probably the most dominant of these properties is the gas-phase proton affinity or deprotonation energy of the proton donor and acceptor groups, which reflects their intrinsic basicity in the absence of any solvent effects. A systematic, detailed study of the effect of excited state electronic and geometric rearrangement on the proton affinities of simple groups might be expected to shed light on the factors that control excited state proton transfer. One such set of molecules are those that contain doubly bonded $\mathrm{C}, \mathrm{N}$, and $\mathrm{O}$ atoms in a planar unsaturated group, many of which are known chromophores. ${ }^{9}$

Several detailed calculations of accurate vertical and adiabatic transition energies for both valence and Rydberg excited states of ethylene ${ }^{5,10,11,15}$ and formaldehyde ${ }^{5,12-16}$ have been recently performed. Early experimental work, reviewed by Merer and Mulliken, ${ }^{17}$ identified five important states in ethylene: ${ }^{1} A_{1}$ (ground state), ${ }^{3} B_{1 u}$ (triplet $\pi \rightarrow \pi^{*}$ state), ${ }^{3} B_{3 u}$ (triplet $\pi \rightarrow 3 s$ Rydberg state), ${ }^{1} B_{3 u}$ (singlet $\pi \rightarrow 3 s$ ), and ${ }^{1} B_{1 u}$ (singlet $\pi \rightarrow \pi^{*}$ ), or $N$ (normal), $T$ (lowest triplet), $T_{R}$ (triplet Rydberg), $R$ (singlet Rydberg), and $V$ (lowest singlet), respectively, in Mulliken's notation. CIS calculations by Foresman et al. ${ }^{5}$ showed the two $\pi \rightarrow \pi^{*}$ states are both stabilized by torsional "twisting" from the planar $D_{2 h}$ structure to a $D_{2 d}$ structure with the two $\mathrm{CH}_{2}$ planes oriented $90^{\circ}$ with respect to each other, along with a lengthening of the $\mathrm{CC}$ bond.

Formaldehyde, the simplest in the class of carbonyl compounds, shows both $\pi \rightarrow \pi^{*}$ and $n \rightarrow \pi^{*}$ transitions, important for photochemical reactions, due to the presence of both the $\pi$ system and two oxygen lone pairs. Hadad et al. ${ }^{14}$ calculated the vertical transition energies for the first 20 singlet states and 10 triplet states. The adiabatic $\pi \rightarrow \pi^{*}$ singlet state is planar, but with a longer $\mathrm{CO}$ bond length, and the triplet $\pi \rightarrow \pi^{*}$ and $n_{y} \rightarrow \pi^{*}$ states distort to a nonplanar geometry. While the second highest occupied molecular orbital (labeled $\pi$ ) is essentially a localized $\pi$ bond, the HOMO (labeled $n_{y}$ ) was found to be not simply a nonbonding oxygen lone pair as stated previously, ${ }^{18}$ but actually delocalized over the entire molecule. However, charge density difference plots showed several pairs of excitations with the same symmetry to be mixtures of $\pi \rightarrow \pi^{*}$ and $n \rightarrow 3 p$ character, requiring some states to have more than one label. The molecular orbitals of acetaldehyde, with just an additional methyl group, were found to have an even greater amount of mixing, such that labels " $\pi$ '" and " $n$ "' no longer fully describe the character of the state (but were used for consistency with earlier work).

In addition to the comprehensive body of work on $\mathrm{H}_{2} \mathrm{C}=\mathrm{CH}_{2}$ and $\mathrm{H}_{2} \mathrm{C}=\mathrm{O}$, the energetics of other selected doubly bonded species isoelectronic with ethylene have been studied. Ground state proton affinities have been calculated for the ethylene anion, $\mathrm{H}_{2} \mathrm{C}=\mathrm{CH}^{-},{ }^{19-21}$ formaldehyde, ${ }^{20-28}$ and $\mathrm{H}_{2} \mathrm{C}=\mathrm{NH}^{20,23,29}$ semiempirically, ${ }^{22,25,29}$ at various levels 


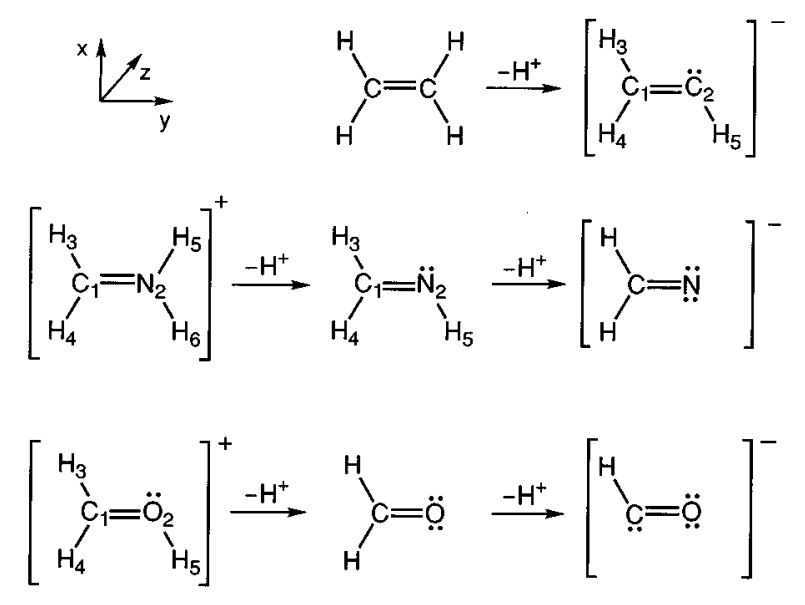

FIG. 1. Deprotonation reactions of doubly bonded species considered in this study.

of $a b$ initio theory, ${ }^{19,23,24,26-28}$ and using several density functional methods. ${ }^{27}$

Calculations by Marudarajan and Scheiner on the ground and excited states of $\mathrm{HC} \equiv \mathrm{CH}^{30}$ and $\mathrm{HC} \equiv \mathrm{N}^{31}$ and their deprotonated anions at the unrestricted Hartree-Fock (UHF) and unrestricted Møller-Plesset (UMP2) level showed that these species undergo a radical change in geometry upon excitation to low-lying excited states, from linear to bent. The lower energy of the acetylene $\pi \rightarrow \pi^{*}$ bent conformation was explained using the molecular orbital (MO) atomic orbital coefficients; the $\pi^{*}$ MO, whose greatest contribution is from the hydrogens, is stabilized by moving these atoms to either the trans or cis position. The excited states of $\mathrm{HC} \equiv \mathrm{N}$ require several $\mathrm{kcal} / \mathrm{mol}$ more energy to deprotonate than the ground state, consistent with a shift in electron density toward the carbon center. However, despite a strong shift in density from $\mathrm{C}$ to $\mathrm{H}$, the deprotonation energies of the first singlet and first two triplet states of acetylene are nearly the same as for the ground state. For both molecules, the difference in the deprotonation energies is less than $5 \%$ of the ground state value. It is not clear from these two examples whether this is a general phenomenon, or peculiar to simple triply bonded molecules.

The current paper reports the study, at a satisfactory $a b$ initio level of theory, of the ground and low-lying excited states of a subset of neutral doubly bonded species $\mathrm{H}_{2} \mathrm{C}=\mathrm{XH}_{n}(\mathrm{X}=\mathrm{C}, \mathrm{N}, \mathrm{O} ; n=2,1,0)$ isoelectronic with ethylene, and the cations and anions that result from protonation and deprotonation, respectively. The relevant deprotonation reactions are shown in Fig. 1. We wish to determine the generality of the isolated results of previous studies of these species, as well as to determine trends in proton affinities based on the character of the state as the electronegativity of the $\mathrm{X}$ atom increases and $\mathrm{XH}$ bonds are replaced with lone pairs. This set of molecules was chosen because the ground state is well described by one localized set of Lewis-type bonding orbitals, and the ground state planar geometry allows clear separation between the $\sigma$ and $\pi$ systems to be retained in the vertically excited states.
In both $\mathrm{HC} \equiv \mathrm{CH}$ and $\mathrm{HC} \equiv \mathrm{N}$, the low-lying valence excited states could be easily correlated between the protonated and deprotonated species because the states were spread fairly far apart and had unique symmetry. This may not usually be the case, and a more systematic method of correlating excited states of similar character is needed. Natural orbital analysis $(\mathrm{NBO})^{32,33}$ is used here to assign the character of each excited state by describing the density in terms of a set of easily recognizable Lewis-type bonding orbitals. This process allows corresponding excited state energies in the protonated and deprotonated species to be "matched" to yield deprotonation energies even in species with a high density of electronic states. We investigate the generality of the geometry changes observed for the adiabatic excited states of ethylene and formaldehyde in states of similar character over the $\mathrm{H}_{2} \mathrm{C}=\mathrm{XH}_{n}$ isoelectronic series, and suggest the origin of these changes based on the excited state character and the orbitals affected by the excitation.

\section{METHOD}

For consistency, the coordinates for all molecules in this study were transformed as indicated in Fig. 1. The $z$ axis is perpendicular to the plane of the molecule, $x$ is in-plane perpendicular to the $\mathrm{C}-\mathrm{X}$ bond, and the $y$ axis is parallel to the $\mathrm{C}-\mathrm{X}$ bond. All calculations were performed using the GAUSSIAN $92^{34}$ electronic structure package. The 6-31 $+\mathrm{G}(d, p)$ basis set ${ }^{35}$ was chosen as the smallest basis set that includes both polarization functions on all atoms ( $3 d$ orbitals on $\mathrm{C}, \mathrm{N}, \mathrm{O} ; 2 p$ on $\mathrm{H}$ ) and diffuse $s p$ functions on heavy atoms, needed to properly describe anions, ${ }^{23,36}$ certain Rydberg excited states, ${ }^{5,14}$ and proton affinities of neutral species with nonbonding electron pairs. ${ }^{37}$ This basis has also been shown to have a particularly low basis set superposition error (BSSE) among available standard basis sets. ${ }^{38,39}$ McMurchie and Davidson, ${ }^{40}$ who used a similar basis for calculations on ethylene, commented that the use of an even more complete basis would clutter the $\pi^{*}$ subspace, as well as obscure identifiable contributions from $\sigma^{*}$ valence orbitals. Indeed, despite yielding more accurate vertical transition energies, many excited states could not be optimized at the 6-311(2+) $\mathrm{G}^{* *}$ level because of multiple curve crossings by the larger manifold of Rydberg states during the relaxation process. The $6-31+\mathrm{G}(d, p)$ basis was the largest one which allowed clear optimization of the valence states.

The ground state geometries were optimized at the restricted Hartree-Fock (RHF) level of theory. Energies of the first 10 singlet and triplet vertical transitions for cations and neutral species (which includes the most important valence and Rydberg states) and the first 15 transitions for anions (or those with transition energies up to $\sim 200 \mathrm{kcal} / \mathrm{mol}$ ) were then computed by the CIS (configuration interaction among all single excitations) method. ${ }^{5,15,41}$ The excited states were next allowed to relax to their optimized geometry at the CIS level, yielding an adiabatic excitation energy. 
The CIS method has been successfully applied to the excited states of a variety of molecules ${ }^{42}$ including the ethylene and formaldehyde studies mentioned above. ${ }^{5,10,14}$ Even with a basis containing diffuse functions, the excited state transition energies tend to be consistently too high by a few $\mathrm{kcal} / \mathrm{mol}$ for valence states, and by as much as $23 \mathrm{kcal} / \mathrm{mol}$ for Rydberg states. Applying Møller-Plesset second-order perturbations (MP2) to the CIS wave function brought the excited state energies of formaldehyde more in line with experiment.

Our analysis of excited state densities showed that while atomic orbitals based on the CIS generalized density have reasonable occupancies and overlap matrix elements, densities from both the CIS-MP2 and spin unrestricted form of the CIS wave function (UCIS) yield unreliable populations. The occupancies of several of the atomic orbitals (AOs) formed from these densities are negative, and overlaps of the resulting LCAO bond orbitals with one another are several times larger than for ground state densities. The occupancyweighted symmetric orthogonalization of these AOs to form natural bond orbitals ${ }^{32}$ yields populations significantly outside the expected range of $0-2$ electrons, ${ }^{43,44}$ making the assignment of excited state character based on such natural bond orbitals (NBOs) unreliable for the purpose of correlating states between protonated and deprotonated species. To avoid this problem we have consistently retained the CIS level to ascertain qualitative and quantitative trends.

Natural bond orbital (NBO) analysis of the ground state and excited state densities was performed separately in order to show the shift in electron density upon excitation, independent of the choice of ground state orbitals. NBOs are the localized set of easily recognizable Lewis-like ( $\sigma$ and $\pi$ bond, lone pair, and core) and non-Lewis ( $\sigma^{*}$ and $\pi^{*}$ antibond and Rydberg) orbitals which are optimal in the sense of orthonormality and maximum occupancy of the Lewis set. The total non-Lewis density $\left(\rho^{*}\right)$, or sum of occupancies of the non-Lewis orbitals, is a measure of the degree of delocalization. There is also a clear distinction between valence and Rydberg orbitals, so that the character can be easily seen in the orbital labels and occupancies, and from the total Rydberg occupancy. If both the occupied and unoccupied MOs involved in the excitation are localized on one or two centers, e.g. for $\pi \rightarrow \pi^{*}$ excitation, then the NBOs not involved in the excitation can be expected to be nearly identical in shape and occupancy to the corresponding ground state orbitals, and only the two corresponding NBOs should be of different form and with depleted bond occupancy and increased antibond occupancy. If the excitation involves MOs with density on several centers, then the optimal set of occupied (Lewis) NBOs will be somewhat different in shape and of lower occupancy to reflect the depletion of density in the bonding regions, while several non-Lewis orbitals will have higher occupancy. Therefore analysis of the differences between the two sets of NBOs shows directly the extent to which the excitation is delocalized.

\section{EXCITED STATE DENSITIES AND DEPROTONATION ENERGIES}

\section{A. Ethylene}

Table I lists the most important singlet and triplet valence and Rydberg excited states of ethylene along with the corresponding transition energies. We consider first the vertical transitions. Each state is characterized by both the symmetry of the transition and the corresponding molecular orbitals as characterized by changes in NBO populations. (Mulliken's labels for the $T, V, T_{R}$, and $R$ states are also indicated.) Included in the label are all NBOs which are populated or depopulated by at least $0.1 e$ compared with the ground state NBOs, in decreasing order of the magnitude of the change in occupancy. Most of the ethylene excitations are fairly localized, involving significant changes to only 2-4 NBOs. ${ }^{45}$ For ethylene, the HOMO is essentially the $\pi_{\mathrm{CC}}$ bond; hence most of the low-lying singlet and triplet states depopulate the $\pi_{\mathrm{CC}} \mathrm{NBO}$. The second highest occupied MO consists of a linear combination of the $\sigma_{\mathrm{CC}}$ and $\sigma_{\mathrm{CH}}$ bonds; states which excite from this MO have weaker $\sigma_{\mathrm{CH}} \mathrm{NBOs}$ with an occupancy of only 1.74-1.84 e compared to 1.987 for the ground state. For the ninth singlet and triplet states, a $\sigma_{\mathrm{CC}}$ orbital with $1.56-1.60 e$, depleted from 1.997, is also involved.

CIS calculations using a basis set with two sets of diffuse functions $\left[6-311(2+) \mathrm{G}^{*}\right]$ were performed on ethylene by Wiberg et al. ${ }^{10}$ In contrast to our NBO analysis, charge density difference plots were used to assign the character of the first 20 singlet and 10 triplet states of ethylene. Accounting for the axis rotation from $C_{2 v}$ to $C_{s}$ symmetry $(x \rightarrow z, y \rightarrow x, z \rightarrow y)$, our ordering and assignments of the valence and $\pi \rightarrow r_{3 s}$ and $\pi \rightarrow r_{3 p}$ Rydberg states are fairly close to theirs (though slightly higher in energy), listed in the fifth column of Table I. However, they assigned the $2{ }^{1} B_{1 g}$ and $2{ }^{3} B_{1 g}$ states as $\pi_{\mathrm{CH}_{2}}^{\prime} \rightarrow \pi^{*}$, whereas we find that these states clearly depopulate the $\sigma_{\mathrm{CH}}$ and $\sigma_{\mathrm{CC}}$ orbitals, and the MO has no $\pi$ character. The only exception in ordering is the energy reversal of the $1^{1} B_{1 u}$ and $1{ }^{1} B_{1 g}$ states, although their two states lie within $1 \mathrm{kcal} / \mathrm{mol}$ of one another. The singlet states were found by Wiberg et al. to be more diffuse and higher in energy than the triplet states of the same character, which also agrees with our results. They also found a significant decrease in CC bond order for the $\pi \rightarrow \pi^{*}$ and Rydberg states, consistent with weakening of the $\pi$ bond.

Listed in the sixth column of Table I are the changes in the natural atomic charges ${ }^{32(c)}$ of the carbon atoms upon excitation. The sixth and ninth singlet and triplet states manifest a noticeable charge shift away from $\mathrm{H}$ and toward $\mathrm{C}$, consistent with depopulation of the $\mathrm{CH}$ bonds. This finding is understandable in light of the shift out of the $\sigma_{\mathrm{CH}} \mathrm{NBOs}$ and into $\pi_{\mathrm{CC}}^{*}$ or a C Rydberg orbital. The NBO analysis of both the $1{ }^{1} B_{1 u}(V)$ and $2{ }^{1} A_{g}$ singlet states confirms significant mixing of $\pi^{*}$ and Rydberg character, previously found by Buenker and Peyerimhoff, ${ }^{46}$ Robin, ${ }^{47}$ and Mulliken. ${ }^{48}$

The excited states of ethylene can be compared with those of its deprotonated form, $\mathrm{CH}_{2} \mathrm{CH}^{-}$, reported in Table II. One $\mathrm{CH}$ bond of ethylene has been replaced with a more 
TABLE I. Vertical excitation energies (VEE in $\mathrm{kcal} / \mathrm{mol}$ ), natural charge shifts, adiabatic excitation energies (AEE), and optimized geometries ${ }^{\mathrm{a}}$ for ethylene.

\begin{tabular}{|c|c|c|c|c|c|c|c|c|c|}
\hline State & Symmetry ${ }^{\mathrm{b}}$ & Transition $^{\mathrm{c}}$ & $\mathrm{VEE}^{\mathrm{d}}$ & Wiberg $^{\mathrm{e}}$ & $\Delta q_{c}{ }^{\mathrm{f}}$ & AEE & $r_{\mathrm{CC}}$ & $r_{\mathrm{CH}}$ & $\theta_{\mathrm{CCH}}$ \\
\hline \multicolumn{10}{|c|}{ Ground state } \\
\hline 0 & $1^{1} A_{g}(N)$ & & & & & & 1.3206 & 1.0764 & 121.66 \\
\hline \multicolumn{10}{|c|}{ Singlet states } \\
\hline 1 & ${ }^{1} B_{3 u}(R)$ & $\pi_{\mathrm{CC}} \rightarrow r_{\mathrm{C}(3 s)}(2)$ & 179.7 & 164.4 & +0.037 & 174.0 & 1.4121 & 1.0714 & 120.31 \\
\hline $2^{\mathrm{g}}$ & $1^{1} B_{1 u}(V)$ & $\pi_{\mathrm{CC}} \rightarrow \pi_{\mathrm{CC}}^{*} r_{\mathrm{C}\left(3 p_{-}\right)}(2)$ & 182.4 & 178.5 & -0.024 & 130.4 & 1.3747 & 1.0897 & 123.64 \\
\hline 3 & $1{ }^{1} B_{1 g}$ & $\pi_{\mathrm{CC}} \rightarrow r_{\mathrm{C}\left(3 p_{x}\right)}(2)$ & 189.9 & 177.8 & +0.068 & 185.6 & 1.3983 & 1.0784 & 119.36 \\
\hline 4 & $1^{1} B_{2 g}$ & $\pi_{\mathrm{CC}} \rightarrow r_{\mathrm{C}\left(3 p_{v}\right)}(2)$ & 192.7 & 181.3 & -0.014 & 185.3 & 1.4297 & 1.0742 & 121.46 \\
\hline $5^{\mathrm{h}}$ & $2{ }^{1} A_{g}$ & $\pi_{\mathrm{CC}} \rightarrow r_{\mathrm{C}\left(3 p_{z}\right)}(2) / \pi_{\mathrm{CC}}^{*}$ & 209.2 & 186.6 & -0.057 & 203.3 & 1.3753 & 1.0779 & 120.60 \\
\hline 6 & $2{ }^{1} B_{1 g}^{g}$ & $\sigma_{\mathrm{CH}}(4) \rightarrow \pi_{\mathrm{CC}}^{*}$ & 216.9 & 214.0 & -0.301 & & \multicolumn{3}{|c|}{ unresolvable curve crossing } \\
\hline 9 & $2{ }^{1} B_{2 g}$ & $\sigma_{\mathrm{CC}} / \sigma_{\mathrm{CH}}(4) \rightarrow \pi_{\mathrm{CC}}^{*}$ & 242.1 & 236.6 & -0.140 & 195.7 & 1.6330 & 1.0676 & 108.30 \\
\hline \multicolumn{10}{|c|}{ Triplet states } \\
\hline $1^{\mathrm{i}}$ & $1^{3} B_{1 u}(T)$ & $\pi_{\mathrm{CC}} \rightarrow \pi_{\mathrm{CC}}^{*}$ & 85.8 & 82.3 & +0.070 & 49.6 & 1.4605 & 1.0762 & 121.26 \\
\hline 2 & $1{ }^{3} B_{3 u}\left(T_{R}\right)$ & $\pi_{\mathrm{CC}} \rightarrow r_{\mathrm{C}(3 s)}(2)$ & 170.5 & 159.3 & +0.021 & 163.9 & 1.4182 & 1.0699 & 120.06 \\
\hline 3 & $1{ }^{3} B_{1 g}$ & $\pi_{\mathrm{CC}} \rightarrow r_{\mathrm{C}\left(3 p_{x}\right)}(2)$ & 186.6 & 175.9 & +0.085 & 182.6 & 1.3941 & 1.0817 & 119.54 \\
\hline 4 & $1{ }^{3} B_{2 g}$ & $\pi_{\mathrm{CC}} \rightarrow r_{\mathrm{C}\left(3 p_{v}\right)}(2)$ & 188.6 & 178.7 & +0.008 & 179.8 & 1.4498 & 1.0806 & 121.71 \\
\hline 5 & $1^{3} A_{g}$ & $\pi_{\mathrm{CC}} \rightarrow r_{\mathrm{C}\left(3 p_{-}\right)}(2)$ & 191.4 & 179.2 & -0.078 & 186.8 & 1.4007 & 1.0706 & 120.38 \\
\hline 6 & $2{ }^{3} B_{1 g}$ & $\sigma_{\mathrm{CC}} / \sigma_{\mathrm{CH}}(4) \rightarrow \pi_{\mathrm{CC}}^{*}$ & 201.2 & 197.4 & -0.300 & & \multicolumn{3}{|c|}{ Unresolvable curve crossing } \\
\hline $9^{j}$ & $2{ }^{3} B_{2 g}$ & $\sigma_{\mathrm{CH}}(4) / \sigma_{\mathrm{CC}} \rightarrow \pi_{\mathrm{CC}}^{*}$ & 225.2 & & -0.151 & 170.1 & 1.4638 & 1.1191 & 87.29 \\
\hline & & & & & & & & 1.0753 & 129.46 \\
\hline
\end{tabular}

${ }^{\mathrm{a} D i s t a n c e s}$ are in angstroms, and angles are in degrees.

${ }^{\mathrm{b}}$ State symmetry in ground state geometry.

${ }^{\mathrm{c}}$ Numbers in parentheses indicate number of such orbitals involved, e.g., orbitals on both $\mathrm{C}$ atoms.

${ }^{\mathrm{d}}$ This work.

${ }^{\mathrm{e}}$ Reference 10, CIS/6-311(2+)G**.

${ }^{\mathrm{f}} \Delta q_{\mathrm{H}}=-1 / 2 \Delta q_{\mathrm{C}}$.

${ }^{\mathrm{g}} \phi_{\mathrm{H}_{5} \mathrm{CCH}_{3}}=88.40^{\circ}$ (" "twist"' dihedral angle, degrees from planarity).

${ }^{\mathrm{h}} \phi_{\mathrm{H}_{5} \mathrm{CCH}_{3}}=33.32^{\circ}$.

${ }^{\mathrm{i}} \phi_{\mathrm{H}_{5} \mathrm{CCH}_{3}}=85.70^{\circ}$.

${ }^{\mathrm{j}} \phi_{\mathrm{H}_{5} \mathrm{CCH}_{3}}=16.75^{\circ}, \phi_{\mathrm{H}_{4} \mathrm{CCH}_{3}}=54.62^{\circ}$ (" "pyramidal distortion" dihedral angle, degrees from planarity).

flexible (and higher energy) carbon lone pair, which becomes the primary contributor to the HOMO. Lone pair NBOs are labeled italic $n$, which should not be confused with nonbonding orbitals (n) which refer to MO types. The first excited singlet state shows evidence of valence-Rydberg mixing similar to that in some of the $\pi \rightarrow \pi^{*}$ excitations in ethylene. The anion's transition energies are lower and closer together than in the neutral.

The nonequivalence of the two $\mathrm{C}$ atoms in $\mathrm{CH}_{2} \mathrm{CH}^{-}$ leads to substantial shifts of density from one to the other. The $n_{\mathrm{C}_{2}} \mathrm{NBO}$ is of course heavily localized on $\mathrm{C}_{2}$, the carbon bonded to one hydrogen. Hence those states exciting out of this orbital tend to shift density from $\mathrm{C}_{2}$ toward $\mathrm{C}_{1}$. The first triplet, for example, shows charge shifts on $\mathrm{C}_{1}$ and $\mathrm{C}_{2}$ in excess of 0.3. (The hydrogens are also affected, but to a lesser extent.) Though the ground state $\mathrm{C}-\mathrm{C} \pi$ bond is polarized $64 \% / 36 \%$ toward $\mathrm{C}_{1}$ (and therefore the $\pi^{*}$ toward $\mathrm{C}_{2}$ ), $\pi$ excitations sometimes also shift charge in the same direction. $\mathrm{C}_{1}$ gains half of an electron in the third singlet. States which show a charge buildup on $\mathrm{C}_{2}$ generally excite into a Rydberg orbital of that atom. Overall, one can conclude that electronic excitation affects charge distribution quite differently in the neutral and anionic forms of ethylene.

Allowing relaxation of the molecular geometries following electronic excitation leads to certain important changes. Table I reports the adiabatic excitation energies (AEE) and optimized geometries for the excited states of ethylene in the last columns. These structures may be compared to the ground state geometry in the first row. Nearly every excitation lengthens the $\mathrm{CC}$ bond. This is not surprising since excitation out of the $\pi$ bond or into the $\pi^{*}$ antibond weakens the $\mathrm{CC}$ bond and causes it to elongate. The only state in which the $\mathrm{CC}$ bond is shorter is the $B_{3 g}$ singlet excited state, which depopulates the $\mathrm{CH}$ bonds and moves density from the hydrogens to the carbons. The $\mathrm{CH}$ bond length is fairly insensitive to excitation except when the $\mathrm{CC}$ bond length undergoes a sizable change. The same is true of the $\mathrm{CCH}$ angle. A longer $r_{\mathrm{CC}}$ tends to shorten $r_{\mathrm{CH}}$ and push the $\mathrm{CH}$ bonds further apart, i.e., $\theta_{\mathrm{CCH}}$ decreases.

The largest increase in bond length is seen in the $2{ }^{1} B_{2 g}$ singlet state, in which the $\sigma_{\mathrm{CC}}$ bond is depopulated in addition to the $\pi_{\mathrm{CC}}^{*}$ occupation. $\pi \rightarrow \pi^{*}$ excitations, as in ${ }^{1} B_{1 u}$ and ${ }^{3} B_{1 u}$, completely sever the $\pi$ bond and the molecule twists out of a planar structure to a staggered conformation nearly of $D_{2 d}$ symmetry (90 twist), shown in Fig. 2(a). Though an electron is excited out of the $\pi$ bond for both states, the fifth singlet transition populates the $\pi^{*}$ orbital to a lesser extent $(0.118 e)$ than the second $(0.921 e)$, and so the torsional twist is less, only $33^{\circ}$ from planar [Fig. 2(b)]. The greatest distortion is seen for the ninth triplet state $\left(\sigma_{\mathrm{CH}} / \sigma_{\mathrm{CC}} \rightarrow \pi^{*}\right)$; the optimized geometry is shown in Fig. 2(c). The $\mathrm{CH}_{2}$ groups are not only twisted relative to each 
TABLE II. Vertical excitation energies $(\mathrm{kcal} / \mathrm{mol})$, natural charge shifts, ${ }^{a}$ adiabatic excitation energies, and optimized geometries for $\mathrm{CH}_{2} \mathrm{CH}^{-}$.

\begin{tabular}{|c|c|c|c|c|c|c|c|c|}
\hline State & Symmetry & Transition & VEE & $\Delta q_{\mathrm{C}_{1}}$ & $\Delta q_{\mathrm{C}_{2}}$ & $\mathrm{AEE}$ & $r_{\mathrm{CC}}$ & $r_{\mathrm{CH}_{5}}$ \\
\hline \multicolumn{9}{|c|}{ Ground state } \\
\hline 0 & $1^{1} A^{\prime}$ & & & & & & 1.3544 & 1.1062 \\
\hline \multicolumn{9}{|c|}{ Singlet states } \\
\hline $1^{\mathrm{b}}$ & $1^{1} A^{\prime \prime}$ & $n_{\mathrm{C}_{2}} \rightarrow \pi_{\mathrm{CC}}^{*} r_{\mathrm{C}_{1}\left(3 p_{z}\right)} / r_{\mathrm{C}_{2}\left(3 p_{z}\right)}$ & 92.7 & -0.331 & +0.132 & 65.1 & 1.3975 & 1.0873 \\
\hline 2 & $2{ }^{1} A^{\prime}$ & $n_{\mathrm{C}_{2}} \rightarrow r_{\mathrm{C}_{2}(3 s p}$ & 99.2 & -0.044 & -0.087 & 74.6 & 1.3938 & 1.0661 \\
\hline 3 & $3^{1} A^{\prime}$ & $n_{\mathrm{C}_{2}} \rightarrow r_{\mathrm{C}_{1}\left(3 s p_{y}\right)} / r_{\mathrm{C}_{2}\left(3 s p_{y}\right)}$ & 105.6 & -0.502 & +0.233 & 87.2 & 1.2754 & 1.0681 \\
\hline 4 & $2{ }^{1} A^{\prime \prime}$ & $\pi_{\mathrm{CC}} \rightarrow r_{\mathrm{C}_{1}\left(3 s p_{y}\right)}$ & 114.5 & -0.260 & +0.006 & 109.0 & 1.4515 & 1.0955 \\
\hline 5 & $4{ }^{1} A^{\prime}$ & $n_{\mathrm{C}_{2}} \rightarrow r_{\mathrm{C}_{1}\left(3 p_{x}\right)} / r_{\mathrm{C}_{2}\left(3 p_{x}\right)}$ & 119.1 & -0.389 & +0.201 & \multicolumn{3}{|c|}{ Unresolvable curve crossing } \\
\hline 6 & $3{ }^{1} A^{\prime \prime}$ & $n_{\mathrm{C}_{2}} \rightarrow r_{\mathrm{C}_{1}\left(3 p_{z}\right)} / r_{\mathrm{C}_{2}\left(3 p_{z}\right)}$ & 120.6 & -0.135 & -0.115 & 103.1 & 1.2780 & 1.0684 \\
\hline 7 & $5^{1} A^{\prime}$ & $\pi_{\mathrm{CC}} \rightarrow r_{\mathrm{C}_{1}\left(3 p_{x}\right)} / r_{\mathrm{C}_{2}\left(3 p_{-}\right)}$ & 131.6 & -0.140 & +0.167 & 103.9 & 1.2767 & 1.0724 \\
\hline 8 & $6{ }^{1} A^{\prime}$ & $n_{\mathrm{C}_{2}} / \pi_{\mathrm{CC}} / \sigma_{\mathrm{CH}_{5}} \rightarrow r_{\mathrm{C}_{2}\left(3 p_{x}\right)} / r_{\mathrm{C}_{1}\left(3 p_{z}\right)} / r_{\mathrm{C}_{1}\left(3 p_{x}\right)}$ & 136.1 & -0.228 & +0.082 & 131.0 & 1.3287 & 1.0786 \\
\hline 11 & $8^{1} A^{\prime}$ & $\pi_{\mathrm{CC}} / n_{\mathrm{C}_{2}} \rightarrow r_{\mathrm{C}_{1}\left(3 p_{2}\right)} / r_{\mathrm{C}_{2}\left(3 p_{2}\right)} / \pi_{\mathrm{CC}}^{*}$ & 150.0 & -0.090 & -0.008 & 147.4 & 1.4028 & 1.0897 \\
\hline 12 & $5{ }^{1} A^{\prime \prime}$ & $\pi_{\mathrm{CC}} / n_{\mathrm{C}_{2}} \rightarrow \pi_{\mathrm{CC}}^{*} / r_{\mathrm{C}_{1}\left(3 p_{-}\right)}^{*} / r_{\mathrm{C}_{2}\left(3 p_{z}\right)}$ & 161.2 & -0.072 & +0.011 & 160.4 & 1.3800 & 1.0949 \\
\hline 13 & $6{ }^{1} A^{\prime \prime}$ & $\pi_{\mathrm{CC}} \rightarrow r_{\mathrm{C}_{2}\left(3 p_{x y}\right)} / r_{\mathrm{C}_{1}\left(3 s p_{x y}\right)}$ & 166.3 & +0.067 & -0.123 & 160.7 & 1.4543 & 1.0956 \\
\hline \multicolumn{9}{|c|}{ Triplet states } \\
\hline $1^{\mathrm{c}}$ & $1^{3} A^{\prime \prime}$ & $n_{\mathrm{C}_{2}} \rightarrow \pi_{\mathrm{CC}}^{*}$ & 65.1 & -0.440 & +0.320 & 41.1 & 1.4518 & 1.0970 \\
\hline 2 & $1^{3} A^{\prime}$ & $n_{\mathrm{C}_{2}} \rightarrow r_{\mathrm{C}_{2}(3 s)}$ & 82.6 & +0.035 & -0.225 & 66.8 & 1.2978 & 1.0596 \\
\hline $3^{\mathrm{c}}$ & $2^{3} A^{\prime \prime}$ & $\pi_{\mathrm{CC}} \rightarrow \pi_{\mathrm{CC}}^{*}$ & 88.6 & +0.376 & -0.309 & 41.1 & 1.4518 & 1.0970 \\
\hline 4 & $2^{3} A^{\prime}$ & $n_{\mathrm{C}_{2}} \rightarrow r_{\mathrm{C}_{1}\left(3 s p_{x v}\right)} / r_{\mathrm{C}_{2}\left(3 s p_{v y}\right)} / \sigma_{\mathrm{CH}_{5}}^{*}$ & 101.4 & -0.355 & +0.151 & 83.3 & 1.2739 & 1.0726 \\
\hline 5 & $3^{3} A^{\prime}$ & $n_{\mathrm{C}_{2}} \rightarrow r_{\mathrm{C}_{2}\left(3 p_{x}\right)} / r_{\mathrm{C}_{1}\left(3 s p_{x y}\right)} / \sigma_{\mathrm{CH}_{5}}^{*}$ & 104.1 & -0.106 & -0.107 & 66.8 & 1.2978 & 1.0596 \\
\hline 6 & $4{ }^{3} A^{\prime}$ & $\pi_{\mathrm{CC}} \rightarrow r_{\mathrm{C}_{1}\left(3 s p_{y}\right)}$ & 108.5 & -0.271 & +0.279 & 83.3 & 1.2739 & 1.0726 \\
\hline 7 & $3^{3} A^{\prime \prime}$ & $n_{\mathrm{C}_{2}} \rightarrow r_{\mathrm{C}_{1}\left(3 p_{z}\right)} / r_{\mathrm{C}_{2}\left(3 p_{z}\right)}$ & 115.5 & -0.052 & -0.183 & 98.7 & 1.2806 & 1.0675 \\
\hline $8^{\mathrm{d}}$ & $5^{3} A^{\prime}$ & $\pi_{\mathrm{CC}} \rightarrow r_{\mathrm{C}_{1}\left(3 p_{z}\right)} / r_{\mathrm{C}_{2}\left(3 p_{z}\right)} / \pi_{\mathrm{CC}}^{*}$ & 126.9 & -0.321 & +0.206 & 123.1 & 1.4297 & 1.0917 \\
\hline 9 & $6^{3} A^{\prime}$ & $\pi_{\mathrm{CC}} \rightarrow r_{\mathrm{C}_{1}\left(3 p_{x y}\right)} / r_{\mathrm{C}_{2}\left(3 s p_{x}\right)}$ & 127.4 & -0.132 & +0.139 & 98.7 & 1.2806 & 1.0675 \\
\hline 13 & $5^{3} A^{\prime \prime}$ & $\pi_{\mathrm{CC}} / n_{\mathrm{C}_{2}} \rightarrow r_{\mathrm{C}_{2}\left(3 p_{y}\right)} / \pi_{\mathrm{CC}}^{*} / r_{\mathrm{C}_{1}\left(3 p_{y}\right)} / r_{\mathrm{C}_{2}\left(3 p_{z}\right)}$ & 159.2 & -0.056 & -0.074 & 154.0 & 1.4562 & 1.0883 \\
\hline
\end{tabular}

${ }^{a} \mathrm{C}_{1}$ is bonded to two hydrogens and $\mathrm{C}_{2}$ to one in $\mathrm{CH}_{2} \mathrm{CH}^{-}$.

${ }^{\mathrm{b}} \phi_{\mathrm{H}_{5} \mathrm{CCH}_{3}}=74.06^{\circ}, \phi_{\mathrm{H}_{4} \mathrm{CCH}_{3}}=31.86^{\circ}$.

${ }^{\mathrm{c}} \phi_{\mathrm{H}_{5} \mathrm{CCH}_{3}}=55.37^{\circ}, \phi_{\mathrm{H}_{4} \mathrm{CCH}_{3}}=48.36^{\circ}$

${ }^{\mathrm{d}} \phi_{\mathrm{H}_{5} \mathrm{CCH}_{3}}=1.87^{\circ}, \phi_{\mathrm{H}_{4} \mathrm{CCH}_{3}}=2.10^{\circ}$.

other, but also pyramidalized $55^{\circ}$ away from planar. Nonplanar distortions generally lower the adiabatic excitation energy more than planar geometry changes, leading to a reordering relative to the vertical excitations. CIS-MP2/6-31+ $\mathrm{G}^{*}$ optimization $^{5}$ of the first singlet $\pi \rightarrow 3 s$ $(R)$ state relaxed the geometry to a $D_{2}$ structure twisted by $24^{\circ}$, compared to $37^{\circ}$ for experiment; ${ }^{49}$ the $\mathrm{CC}$ bond also

\section{$\mathrm{CH}_{2} \mathrm{CH}_{2}$}
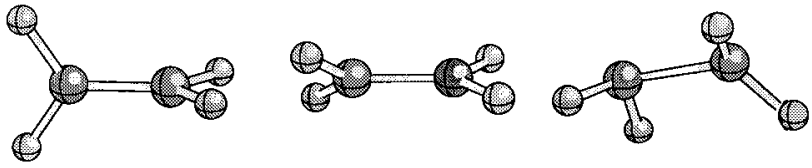

a) singlet $2\left({ }^{1} B_{14}\right)$ triplet $1\left({ }^{3} B_{1 u}\right)$

b) singlet $5\left({ }^{1} A_{g}\right)$

c) triplet $9\left({ }^{3} \mathrm{~B}_{2 g}\right)$
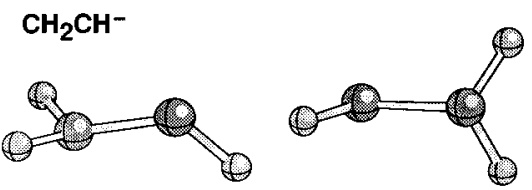

d) singlet $1\left({ }^{1} A^{\prime \prime}\right)$

e) triplet $1\left({ }^{3} A^{\prime \prime}\right)$

FIG. 2. Nonplanar excited state geometries (CIS 6-31+ $\mathrm{G}^{*}$ ) of $\mathrm{CH}_{2} \mathrm{CH}_{2}$ and $\mathrm{CH}_{2} \mathrm{CH}^{-}$.

lengthened to $1.41 \AA$ and the $\mathrm{HCH}$ angle increased by $3.0^{\circ}$. The CIS method without correlation finds a planar optimized geometry for the $\pi \rightarrow r_{\mathrm{C}(3 s)}$ states, though the surface is relatively flat relative to out-of-plane twist. More specifically, the CIS energy of the $R$ state at the CIS/MP2 optimized value of $\phi=23.66^{\circ}$ lies only $0.66 \mathrm{kcal} / \mathrm{mol}$ above that of the planar geometry.

Table II lists the optimized $\mathrm{CC}$ and $\mathrm{C}-\mathrm{H}_{5}$ bond lengths and $\mathrm{HCCH}$ rotation angles for $\mathrm{CH}_{2} \mathrm{CH}^{-}$. Optimized geometries for some excited states could not be found, particularly in states involving changes in the torsion angle. In these cases the given CIS root shows mixing of several occupied and unoccupied MOs, and the optimization procedure cannot follow the excited state across multiple curve crossings. This occurs more often for cations and anions than for neutral species. One also sees more than one state converge to the same geometry when optimization occurs. An example of this is the pair of ${ }^{3} A^{\prime}$ states numbered 2 and 5 . As in the case of ethylene, strong excitation into the $\mathrm{CH}_{2} \mathrm{CH}^{-} \pi^{*}$ antibond often leads to nonplanar distortion of the molecule, as in the first singlet and triplet states [Figs. 2(d) and 2(e)], or a large increase in $\mathrm{CC}$ bond length. However, unlike $\mathrm{CH}_{2} \mathrm{CH}_{2}$ itself the anionic species can relax to relieve steric strain by increasing the $\mathrm{CCH}_{5}$ bond angle from the ground state value of $108.57^{\circ}$ to the more linear $140^{\circ}-149^{\circ}$ observed in many of the Rydberg excited states. 
TABLE III. Vertical excitation energies ( $\mathrm{kcal} / \mathrm{mol})$, adiabatic excitation energies, and optimized geometries for $\mathrm{CH}_{2} \mathrm{NH}_{2}^{+}$.

\begin{tabular}{|c|c|c|c|c|c|c|c|c|c|}
\hline State & Symmetry & Transition & VEE & AEE & $r_{\mathrm{CN}}$ & $r_{\mathrm{CH}}^{\mathrm{a}}$ & $r_{\mathrm{NH}}{ }^{\mathrm{a}}$ & $\theta_{\mathrm{NCH}}$ & $\theta_{\mathrm{HNC}}$ \\
\hline \multicolumn{10}{|c|}{ Ground state } \\
\hline 0 & $1{ }^{1} A_{1}$ & & & & 1.2633 & 1.0761 & 1.0043 & 119.81 & 121.70 \\
\hline \multicolumn{10}{|c|}{ Singlet states } \\
\hline $1^{\mathrm{b}}$ & $1{ }^{1} A_{2}$ & $\pi_{\mathrm{CN}} / \sigma_{\mathrm{CH}}(2) \rightarrow \pi_{\mathrm{CN}}^{*}$ & 206.2 & 114.8 & 1.3165 & 1.0902 & 1.0145 & 121.15 & 122.90 \\
\hline $2^{\mathrm{b}}$ & $2{ }^{1} A_{1}$ & $\pi_{\mathrm{CN}} \rightarrow \pi_{\mathrm{CN}}^{*}$ & 234.4 & 114.8 & 1.3165 & 1.0902 & 1.0145 & 121.15 & 122.90 \\
\hline $3^{b}$ & $1{ }^{1} B_{1}$ & $\sigma_{\mathrm{CN}} / \sigma_{\mathrm{CH}}(2) / \pi_{\mathrm{CN}} / \sigma_{\mathrm{NH}}(2) \rightarrow \pi_{\mathrm{CN}}^{*}$ & 271.2 & 114.8 & 1.3165 & 1.0902 & 1.0145 & 121.15 & 122.90 \\
\hline $4^{b}$ & $2{ }^{1} B_{1}$ & $\pi_{\mathrm{CN}} \rightarrow r_{\mathrm{N}(3 s)} / \sigma_{\mathrm{NH}}^{*}(2)$ & 278.6 & 114.8 & 1.3165 & 1.0902 & 1.0145 & 121.15 & 122.90 \\
\hline 5 & $2{ }^{1} A_{2}$ & $\pi_{\mathrm{CN}} \rightarrow r_{\mathrm{N}\left(3 p_{x}\right)} / \sigma_{\mathrm{NH}}^{*}(2)$ & 298.4 & 280.1 & 1.3255 & 1.0975 & 1.1895 & 120.08 & 126.51 \\
\hline $6^{\mathrm{b}}$ & $1{ }^{1} B_{2}$ & $\sigma_{\mathrm{CH}}(2) / \sigma_{\mathrm{NH}}(2) \rightarrow r_{\mathrm{C}(3 s)} / r_{\mathrm{N}(3 s)} / \sigma_{\mathrm{NH}}^{*}(2) / \sigma_{\mathrm{CH}}^{*}$ & 322.5 & 114.8 & 1.3165 & 1.0902 & 1.0145 & 121.15 & 122.90 \\
\hline $7^{b}$ & $3{ }^{1} B_{1}$ & $\pi_{\mathrm{CN}} \rightarrow r_{\mathrm{C}\left(3 s p_{y}\right)} / r_{\mathrm{N}\left(3 p_{y}\right)} / \sigma_{\mathrm{CH}}^{*}(2)$ & 327.5 & 114.8 & 1.3165 & 1.0902 & 1.0145 & 121.15 & 122.90 \\
\hline 8 & $3{ }^{1} A_{2}$ & $\pi_{\mathrm{CN}} / \sigma_{\mathrm{NH}}(2) \rightarrow \pi_{\mathrm{CN}}^{*}$ & 333.4 & 280.1 & 1.3255 & 1.0975 & 1.1895 & 120.08 & 126.51 \\
\hline $9^{c}$ & $3{ }^{1} A_{1}$ & $\pi_{\mathrm{CN}} \rightarrow r_{\mathrm{N}\left(3 p_{z}\right)} / r_{\mathrm{C}\left(3 p_{z}\right)} / \pi_{\mathrm{CN}}^{*}$ & 340.4 & 318.3 & 1.3026 & 1.1225 & 1.0338 & 118.72 & 121.72 \\
\hline 10 & $2{ }^{1} B_{2}$ & $\sigma_{\mathrm{CH}}(2) / \sigma_{\mathrm{NH}}(2) \rightarrow r_{\mathrm{C}\left(3 p_{z}\right)}$ & 350.3 & & & ciative- & H bonds & ken & \\
\hline \multicolumn{10}{|c|}{ Triplet states } \\
\hline \multirow[t]{2}{*}{$1^{\mathrm{d}}$} & $1^{3} A_{1}$ & $\pi_{\mathrm{CN}} \rightarrow \pi_{\mathrm{CN}}^{*}$ & 124.2 & 92.2 & 1.3658 & 1.0931 & 1.0159 & 110.50 & 124.00 \\
\hline & & & & & & 1.0781 & 1.0142 & 115.95 & 120.42 \\
\hline \multirow[t]{2}{*}{$2^{\mathrm{d}}$} & $1{ }^{3} A_{2}$ & $\pi_{\mathrm{CN}} / \sigma_{\mathrm{CH}}(2) \rightarrow \pi_{\mathrm{CN}}^{*} / \sigma_{\mathrm{CH}}^{*}(2)$ & 183.2 & 92.2 & 1.3658 & 1.0781 & 1.0142 & 115.95 & 120.42 \\
\hline & & & & & & 1.0781 & 1.0142 & 115.95 & 120.42 \\
\hline \multirow[t]{2}{*}{$3^{\mathrm{d}}$} & $1^{3} B_{1}$ & $\pi_{\mathrm{CN}} / \sigma_{\mathrm{CN}} / \sigma_{\mathrm{CH}}(2) \rightarrow \pi_{\mathrm{CN}}^{*}$ & 243.6 & 92.2 & 1.3658 & 1.0781 & 1.0142 & 115.95 & 120.42 \\
\hline & & & & & & 1.0781 & 1.0142 & 115.95 & 120.42 \\
\hline 4 & $2{ }^{3} B_{1}$ & $\pi_{\mathrm{CN}} \rightarrow \sigma_{\mathrm{NH}}^{*}(2) / r_{\mathrm{N}(3 s)}$ & 264.6 & \multicolumn{6}{|c|}{ Dissociative $\mathrm{N}-\mathrm{H}$ bond broken } \\
\hline 5 & $2^{3} A_{2}$ & $\pi_{\mathrm{CN}} \rightarrow \sigma_{\mathrm{NH}}^{*}(2) / r_{\mathrm{N}\left(3 p_{x}\right)}$ & 291.4 & \multicolumn{6}{|c|}{ Dissociative $-\mathrm{N}-\mathrm{H}$ bond broken } \\
\hline 6 & $1{ }^{3} B_{2}$ & $\sigma_{\mathrm{CH}}(2) / \sigma_{\mathrm{NH}}(2) \rightarrow \sigma_{\mathrm{CH}}^{*}(2) / r_{\mathrm{C}\left(3 s p_{y}\right)}$ & 298.2 & \multicolumn{6}{|c|}{ Dissociative $-\mathrm{C}-\mathrm{H}$ bond broken } \\
\hline 7 & $2^{3} A_{1}$ & $\sigma_{\mathrm{CH}}(2) / \sigma_{\mathrm{NH}}(2) \rightarrow \sigma_{\mathrm{CH}}^{*}(2) / \sigma_{\mathrm{NH}}^{*}(2)$ & 309.7 & \multicolumn{6}{|c|}{ Dissociative $-\mathrm{C}-\mathrm{H}$ bond broken } \\
\hline 8 & $3{ }^{3} A_{2}$ & $\pi_{\mathrm{CN}} / \sigma_{\mathrm{NH}}(2) \rightarrow \pi_{\mathrm{CN}}^{*} / \sigma_{\mathrm{NH}}^{*}(2)$ & 318.6 & \multicolumn{6}{|c|}{ Unresolvable curve crossing } \\
\hline \multirow[t]{2}{*}{$9^{\mathrm{d}}$} & $3{ }^{3} B_{1}$ & $\pi_{\mathrm{CN}} \rightarrow r_{\mathrm{C}(3 s)} / r_{\mathrm{N}\left(3 p_{y}\right)} / \sigma_{\mathrm{CH}}^{*}(2)$ & 318.9 & 92.2 & 1.3658 & 1.0931 & 1.0159 & 110.50 & 124.00 \\
\hline & & & & & & 1.0781 & 1.0142 & 115.95 & 120.42 \\
\hline 10 & $2^{3} B_{2}$ & $\sigma_{\mathrm{CH}}(2) / \sigma_{\mathrm{NH}}(2) \rightarrow \sigma_{\mathrm{NH}}^{*}(2) / r_{\mathrm{C}\left(3 p_{y}\right)}$ & 320.5 & \multicolumn{6}{|c|}{ Dissociative $-\mathrm{N}-\mathrm{H}$ bond broken } \\
\hline
\end{tabular}

a Triplet states optimize to $\mathrm{C}_{1}$ geometry; first row $=r_{\mathrm{CH}_{1}}, r_{\mathrm{NH}_{3}}$; second row $=r_{\mathrm{CH}_{2}}, r_{\mathrm{NH}_{4}}$

${ }^{\mathrm{b}} \phi_{\mathrm{H}_{5} \mathrm{NCH}_{4}}=90.00^{\circ}$.

${ }^{\mathrm{c}} \phi_{\mathrm{H}_{5} \mathrm{NCH}_{4}}=56.24^{\circ}$.

${ }^{\mathrm{d}} \phi_{\mathrm{H}_{5} \mathrm{NCH}_{4}}=81.05^{\circ}, \phi_{\mathrm{H}_{4} \mathrm{CNH}_{3}}=48.04^{\circ}$.

\section{B. $\mathrm{CH}_{2} \mathrm{NH}$}

Table III lists the excited states of $\mathrm{CH}_{2} \mathrm{NH}_{2}^{+}$, which has the same basic geometry as ethylene except that one carbon is replaced by the more electronegative N. Like ethylene, most of the excitations come from the $\pi$ HOMO. The lowlying excitations in $\mathrm{CH}_{2} \mathrm{NH}_{2}^{+}$involve $\pi^{*}$, as compared to the Rydberg transitions of $\mathrm{CH}_{2} \mathrm{CH}_{2}$. Compared with ethylene, excitations of $\mathrm{CH}_{2} \mathrm{NH}_{2}^{+}$are less localized, with more of them involving MOs which include the $\mathrm{CH}$ and $\mathrm{NH}$ bonds and antibonds in addition to the $\pi_{\mathrm{CN}}$ or $\pi_{\mathrm{CN}}^{*}$ orbitals. The vertical excitation energies are spread further apart for this cation than for ethylene, with the Rydberg excitations occurring higher in the list relative to the valence excitations.

Table IV shows the excited states of neutral $\mathrm{CH}_{2} \mathrm{NH}$. As in the $\mathrm{CH}_{2} \mathrm{CH}_{2} / \mathrm{CH}_{2} \mathrm{CH}^{-}$case, removal of a proton from $\mathrm{CH}_{2} \mathrm{NH}_{2}^{+}$reduces the spacing of the excited state energy levels. Nearly every excitation shifts electron density from $\mathrm{N}$ to $\mathrm{C}$, similar to $\mathrm{C}_{2} \rightarrow \mathrm{C}_{1}$ shifts in $\mathrm{CH}_{2} \mathrm{CH}^{-}$. The greatest charge shifts from $\mathrm{N}$ to $\mathrm{C}, 0.38-0.53 e$, are seen in excitations from the nitrogen lone pair to a carbon Rydberg orbital. Also significant are excitations into the $\pi^{*}$ orbital, which is polarized toward carbon.

Removal of a second proton to form $\mathrm{CH}_{2} \mathrm{~N}^{-}$produces a further reduction in state separation. Several states of the anion lie less than $100 \mathrm{kcal} / \mathrm{mol}$ above the ground state. This anion, which has the same type of geometry as formaldehyde (vide infra), has two $\mathrm{N}$ lone pairs. The set of Lewis-type NBOs which best describes the ground state density hybridizes the two nitrogen lone pairs as a higher energy $n_{2 p_{x}}$ orbital and the other $n_{2 s p_{y}} ; \mathrm{H}_{2} \mathrm{CO}$ similarly contains the same two lone pairs on $\mathrm{O}$. NBO analysis of states involving excitation out of the lone pairs in both $\mathrm{CH}_{2} \mathrm{~N}^{-}$and $\mathrm{CH}_{2} \mathrm{O}$ show that the HOMO is primarily of $2 p_{x}$ character, and more energy is needed to excite from the more stable lone pair $\mathrm{MO}$ which is of $s p_{y}$ character. In $\mathrm{CH}_{2} \mathrm{NH}$ and $\mathrm{CH}_{2} \mathrm{OH}^{+}$, the single lone pair is of $s p_{x} p_{y}$ character, indicating that a rehybridization occurs upon protonation.

The greater contributions from the $\mathrm{CH}$ and $\mathrm{NH}$ bonds and antibonds in excited state $\mathrm{CH}_{2} \mathrm{NH}_{2}^{+}$(Table III) leads to several states which break one or more of these bonds and dissociate.$^{50}$ The tenth singlet state breaks both $\mathrm{C}-\mathrm{H}$ bonds in a symmetric manner, while the triplet dissociations break the symmetry by dissociating only one of two equivalent bonds. Several of the singlet states optimize to the same twisted $C_{2 d}$ geometry [Fig. 3(a)] due to significant MO mixing, while a reduced $\mathrm{C}_{2}$ triplet excited state geometry with a 
TABLE IV. Vertical excitation energies (kcal/mol), adiabatic excitation energies, optimized geometries, and changes in orbital occupancy for $\mathrm{CH}_{2} \mathrm{NH}$.

\begin{tabular}{|c|c|c|c|c|c|c|c|c|c|}
\hline State & Symmetry & Transition & VEE & AEE & $r_{\mathrm{CN}}$ & $r_{\mathrm{NH}_{5}}$ & $\phi_{\mathrm{H}_{4} \mathrm{CNH}_{3}}$ & $\phi_{\mathrm{H}_{5} \mathrm{NCH}_{3}}$ & $\begin{array}{l}\text { Population } \\
\text { change }^{\mathrm{a}}\end{array}$ \\
\hline \multicolumn{10}{|c|}{ Ground state } \\
\hline 0 & $1^{1} A^{\prime}$ & & & & 1.2522 & 1.0052 & 0.00 & 0.00 & \\
\hline \multicolumn{10}{|c|}{ Singlet states } \\
\hline 1 & $1^{1} A^{\prime \prime}$ & $n_{\mathrm{N}\left(2 p_{x y}\right)} \rightarrow \pi_{\mathrm{CN}}^{*}$ & 135.9 & 94.2 & 1.3165 & 1.0092 & 25.98 & 77.01 & $\Delta \rho\left(\pi^{*}\right)=+0.927$ \\
\hline 2 & $2{ }^{1} A^{\prime}$ & $n_{\mathrm{N}\left(2 p_{x y}\right)} \rightarrow r_{\mathrm{C}\left(3 s p_{y}\right)} / r_{\mathrm{N}(3 s)} / \sigma_{\mathrm{NH}}^{*}$ & 192.2 & 175.1 & 1.2038 & 1.0540 & 0.00 & 0.00 & \\
\hline 3 & $3{ }^{1} A^{\prime}$ & $\pi_{\mathrm{CN}} \rightarrow \pi_{\mathrm{CN}}^{*}$ & 206.7 & 178.8 & 1.5420 & 1.0223 & 45.29 & 48.36 & $\Delta \rho(\pi)=-0.802$ \\
\hline & & & & & & & & & $\Delta \rho\left(\pi^{*}\right)=+0.912$ \\
\hline 4 & $2{ }^{1} A^{\prime \prime}$ & $\pi_{\mathrm{CN}} \rightarrow r_{\mathrm{N}\left(3 s p_{x}\right)} / r_{\mathrm{C}\left(3 s p_{x y}\right)} / \sigma_{\mathrm{NH}}^{*}$ & 209.7 & 200.3 & 1.3548 & 1.0500 & 0.00 & 0.00 & $\Delta \rho(\pi)=-0.993$ \\
\hline 5 & $4{ }^{1} A^{\prime}$ & $n_{\mathrm{N}\left(2 p_{x y}\right)} \rightarrow r_{\mathrm{C}\left(3 s p_{y}\right)}$ & 220.6 & 197.0 & 1.1931 & 1.0495 & 0.00 & 0.00 & \\
\hline 7 & $3{ }^{1} A^{\prime \prime}$ & $\left.\pi_{\mathrm{CN}} \rightarrow r_{\mathrm{C}\left(3 s p_{x y}\right.}\right) / r_{\mathrm{H}_{3}(2 s)} / \sigma_{\mathrm{CH}_{3}}^{*}$ & 233.8 & 208.0 & 1.2802 & 1.0216 & 0.00 & 38.37 & $\Delta \rho(\pi)=-0.735$ \\
\hline 8 & $4{ }^{1} A^{\prime \prime}$ & $n_{\mathrm{N}\left(2 p_{x y}\right)} \rightarrow r_{\mathrm{C}\left(3 p_{z}\right)} / r_{\mathrm{N}\left(3 p_{z}\right)}$ & 235.6 & 215.4 & 1.1862 & 1.0005 & 1.33 & 30.23 & $\begin{aligned} \Delta \rho\left(r_{\mathrm{c}}\right) & =+0.574 \\
\Delta \rho\left(r_{\mathrm{N}}\right) & =+0.225\end{aligned}$ \\
\hline 10 & $6{ }^{1} A^{\prime \prime}$ & $\sigma_{\mathrm{CH}_{3}} / \sigma_{\mathrm{CN}} / \pi_{\mathrm{CN}} / \sigma_{\mathrm{NH}} \rightarrow \pi_{\mathrm{CN}}^{*}$ & 241.9 & 94.2 & 1.3165 & 1.0092 & 25.98 & 77.01 & $\begin{array}{c}\Delta \rho(\pi)=-0.242 \\
\Delta \rho\left(\pi^{*}\right)=+0.667\end{array}$ \\
\hline \multicolumn{10}{|c|}{ Triplet states } \\
\hline 1 & $1^{3} A^{\prime}$ & $\pi_{\mathrm{CN}} \rightarrow \pi_{\mathrm{CN}}^{*}$ & 99.7 & 63.3 & 1.4228 & 1.0119 & 42.32 & 76.56 & $\begin{array}{c}\Delta \rho(\pi)=-0.994 \\
\Delta \rho\left(\pi^{*}\right)=+0.988\end{array}$ \\
\hline 2 & $1^{3} A^{\prime \prime}$ & $n_{\mathrm{N}\left(2 p_{x v}\right)} \rightarrow \pi_{\mathrm{CN}}^{*}$ & 109.5 & 62.8 & 1.4118 & 1.0099 & 44.92 & 30.50 & $\Delta \rho\left(\pi^{*}\right)=+0.959$ \\
\hline 3 & $2^{3} A^{\prime}$ & $n_{\mathrm{N}\left(2 p_{x y}\right)} \rightarrow \sigma_{\mathrm{NH}}^{*} / r_{\mathrm{C}(3 s)} / r_{\mathrm{N}(3 s)}$ & 181.4 & 63.3 & 1.4228 & 1.0119 & 42.32 & 76.56 & \\
\hline 4 & $2^{3} A^{\prime \prime}$ & $\pi_{\mathrm{CN}} \rightarrow r_{\mathrm{C}(3 s)} / r_{\mathrm{N}(3 s)} / \sigma_{\mathrm{NH}}^{*}$ & 202.6 & \multicolumn{6}{|c|}{ Dissociative $-\mathrm{N}-\mathrm{H}$ bond broken } \\
\hline 5 & $3^{3} A^{\prime}$ & $\left.n_{\mathrm{N}\left(2 p_{x y}\right.}\right) / \sigma_{\mathrm{CH}}(2) \rightarrow r_{\mathrm{C}\left(3 s p_{x y}\right)} / \sigma_{\mathrm{CH}_{3}}^{*} / \sigma_{\mathrm{NH}}^{*} / r_{\mathrm{H}_{3}(2 s)}$ & 210.6 & \multicolumn{6}{|c|}{ Dissociative $-\mathrm{C}-\mathrm{H}_{3}$ bond broken } \\
\hline 7 & $3^{3} A^{\prime \prime}$ & $\pi_{\mathrm{CN}} / \sigma_{\mathrm{CH}_{3}} / \sigma_{\mathrm{CN}} / \sigma_{\mathrm{NH}} \rightarrow \pi_{\mathrm{CN}}^{*}$ & 219.9 & 62.8 & 1.4118 & 1.0099 & 44.92 & 30.50 & \\
\hline 8 & $4{ }^{3} A^{\prime \prime}$ & $\pi_{\mathrm{CN}} \rightarrow r_{\mathrm{C}\left(3 p_{x y}\right)} / \sigma_{\mathrm{CH}_{3}}^{*} / r_{\mathrm{H}_{3}(2 s)}$ & 230.5 & 63.3 & 1.4228 & 1.0119 & 42.32 & 76.56 & \\
\hline 10 & $6^{3} A^{\prime \prime}$ & $\pi_{\mathrm{CN}} \rightarrow r_{\mathrm{C}\left(3 p_{x y}\right)} / \pi_{\mathrm{CN}}^{*} / r_{\mathrm{C}\left(3 p_{z}\right)}$ & 238.1 & 63.3 & 1.4228 & 1.0119 & 42.32 & 76.56 & \\
\hline
\end{tabular}

${ }^{\mathrm{a}}$ Change(s) in $\pi, \pi *$, or $r_{\left(3 p_{z}\right)}$ orbital occupancies for states in which they are the major contributors.

significantly longer $\mathrm{CN}$ bond $(1.366 \AA)$ and a $\mathrm{CH}_{2}$ group pyramidalized $48^{\circ}$ out of plane [Fig. 3(c)] appears to be the most stable configuration. In general, higher energy cation Rydberg transitions collapse to the adiabatic geometry of a lower valence excited state.

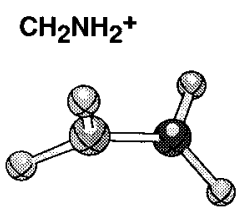

a) singlet $2\left({ }^{1} \mathrm{~A}_{2}\right)$

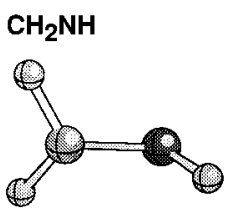

d) singlet $1\left({ }^{1} A^{\prime \prime}\right)$

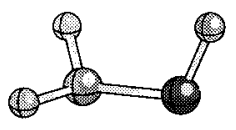

f) triplet $1\left({ }^{3} A^{\prime}\right)$

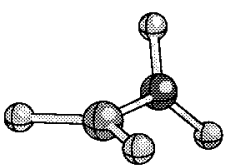

b) singlet $9\left({ }^{1} \mathrm{~A}_{1}\right)$

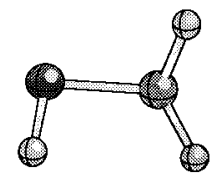

e) singlet $3\left({ }^{A} A^{\prime}\right)$

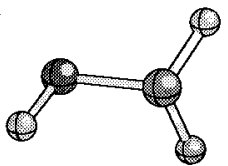

g) triplet $2\left({ }^{3} A^{\prime \prime}\right)$

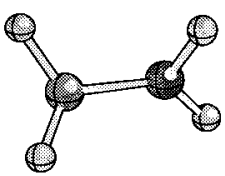

c) triplet $1\left(3 A_{1}\right)$
FIG. 3. Nonplanar excited state optimized geometries (CIS $6-31+\mathrm{G}^{*}$ ) of $\mathrm{CH}_{2} \mathrm{NH}_{2}^{+}$and $\mathrm{CH}_{2} \mathrm{NH}$.
The degree of nonplanarity of the adiabatic excited state geometries of $\mathrm{CH}_{2} \mathrm{NH}$ is described by the dihedral angles in the second and third last columns of Table IV. When the CN bond is weakened by excitation out of the $\pi$ bond or into the $\pi^{*}$ antibond, the NH bond twists out of the plane and the $\mathrm{CH}_{2}$ group pyramidalizes, either in the opposite direction [Figs. 3(d) and 3(g)] or in the same direction [Figs. 3(e) and 3(f)]. The correlation between $\pi, \pi^{*}$, or $r_{3 p_{z}}$ character and the amount of nonplanar distortion can be seen by comparing the two torsion angles with the change in population of the $\pi, \pi^{*}$, or the sum of the $r_{\mathrm{C}\left(3 p_{z}\right)}$ and $r_{\mathrm{N}\left(3 p_{z}\right)}$ populations in the last column of Table IV. For nonplanar geometries this change is at least $0.7 e$. An exception is the fourth singlet state, in which the planar geometry appears to be stabilized by partial population of the $\sigma_{\mathrm{NH}}^{*}$ orbital. For the other states, the greater this change in population, the larger the nonplanar distortion.

Comparison of the excited state optimized geometries of $\mathrm{CH}_{2} \mathrm{~N}^{-}$(Table V) and $\mathrm{CH}_{2} \mathrm{O}$ shows that the $\mathrm{CH}_{2} \mathrm{~N}^{-}$overall negative charge stabilizes the planar geometries relative to nonplanar distortions such that only the triplet $n_{\mathrm{N}\left(2 p_{x}\right)} \rightarrow \pi^{*}$ excitation causes the $\mathrm{CH}$ bonds to bend out of the plane. Because of the higher energy of the $\mathrm{CH}_{2} \mathrm{~N}^{-} \mathrm{HOMO}$, which is primarily the $n_{\mathrm{N}\left(2 p_{x}\right)}$ lone pair, compared to that of $\mathrm{CH}_{2} \mathrm{O}$, which also includes some $\sigma_{\mathrm{CH}}$ and $\pi_{\mathrm{CO}}$ character with the $n_{\mathrm{O}\left(2 p_{x}\right)}$ orbital, an electron is more easily excited from this orbital (Table V). 
TABLE V. Vertical excitation energies (kcal/mol), adiabatic excitation energies, and optimized geometries for $\mathrm{CH}_{2} \mathrm{~N}^{-}$.

\begin{tabular}{|c|c|c|c|c|c|c|c|}
\hline State & Symmetry & Transition & VEE & AEE & $r_{\mathrm{CN}}$ & $r_{\mathrm{CH}}$ & $\theta_{\mathrm{NCH}}$ \\
\hline \multicolumn{8}{|c|}{ Ground state } \\
\hline 0 & $1{ }^{1} A_{1}$ & & & & 1.2406 & 1.1229 & 125.28 \\
\hline \multicolumn{8}{|c|}{ Singlet states } \\
\hline 1 & $1^{1} A_{2}$ & $n_{\mathrm{N}\left(2 p_{r}\right)} \rightarrow \pi_{\mathrm{CN}}^{*} / r_{\mathrm{C}\left(3 p_{z}\right)}$ & 60.1 & 55.4 & 1.3203 & 1.0863 & 121.47 \\
\hline 2 & $1{ }^{1} B_{2}$ & $n_{\mathrm{N}\left(2 p_{x}\right)} \rightarrow r_{\mathrm{C}(3 s)} / r_{\mathrm{N}\left(3 s p_{y}\right)}$ & 76.5 & 73.7 & 1.1983 & 1.1013 & 123.83 \\
\hline 3 & $2{ }^{1} B_{2}$ & $n_{\mathrm{N}\left(2 p_{x}\right)} \rightarrow r_{\mathrm{C}\left(3 p_{y}\right)}$ & 91.2 & 88.9 & 1.1969 & 1.1127 & 126.48 \\
\hline 4 & $2{ }^{1} A_{1}$ & $n_{\mathrm{N}\left(2 p_{x}\right)} \rightarrow r_{\mathrm{C}\left(3 p_{y}\right)}$ & 97.8 & 94.1 & 1.2001 & 1.0946 & 121.75 \\
\hline 5 & $2{ }^{1} A_{2}$ & $n_{\mathrm{N}\left(2 p_{x}\right)} \rightarrow r_{\mathrm{C}\left(3 p_{-}\right)} / r_{\mathrm{N}\left(3 p_{z}\right)}$ & 114.9 & 113.8 & 1.2214 & 1.1020 & 123.45 \\
\hline 6 & $1{ }^{1} B_{1}$ & $\pi_{\mathrm{CN}} \rightarrow r_{\mathrm{C}\left(3 s p_{x}\right)}$ & 142.0 & 132.0 & 1.3506 & 1.0777 & 122.42 \\
\hline 7 & $3{ }^{1} A_{1}$ & $\pi_{\mathrm{CN}} / n_{\mathrm{N}\left(2 p_{x}\right)} \rightarrow r_{\mathrm{C}\left(3 p_{z}\right)} / \pi_{\mathrm{CH}}^{*} / r_{\mathrm{C}\left(3 p_{x}\right)}$ & 153.5 & 145.9 & 1.3401 & 1.0881 & 122.79 \\
\hline 10 & $3{ }^{1} A_{2}$ & $\pi_{\mathrm{CN}} \rightarrow r_{\mathrm{C}\left(3 p_{x}\right)} / \pi_{\mathrm{CN}}^{*}$ & 160.2 & 148.9 & 1.3540 & 1.0764 & 120.27 \\
\hline 12 & $3{ }^{1} B_{1}$ & $n_{\mathrm{N}\left(2 p_{r}\right)} \rightarrow \pi_{\mathrm{CN}}^{*} / \sigma_{\mathrm{CN}}^{*} / r_{\mathrm{C}\left(3 p_{-}\right)}$ & 180.7 & 160.6 & 1.4709 & 1.0734 & 115.07 \\
\hline 14 & $5^{1} A_{1}$ & $\pi_{\mathrm{CN}} / n_{\mathrm{N}\left(2 s p_{y}\right)} \rightarrow \pi_{\mathrm{CN}}^{*} / r_{\mathrm{N}\left(3 p_{z}\right)}$ & 203.6 & 189.9 & 1.3921 & 1.0980 & 122.69 \\
\hline \multicolumn{8}{|c|}{ Triplet states } \\
\hline 1 & $1^{3} A_{2}$ & $n_{\mathrm{N}\left(2 p_{.}\right)} \rightarrow \pi_{\mathrm{CN}}^{*} / r_{\mathrm{C}\left(3 p_{-}\right)}$ & 42.8 & 31.4 & 1.3718 & 1.0976 & 113.11 \\
\hline 2 & $1{ }^{3} B_{2}$ & $n_{\mathrm{N}\left(2 p_{x}\right)} \rightarrow r_{\mathrm{C}(3 s)} / r_{\mathrm{N}\left(3 s p_{y}\right)}$ & 71.6 & 68.8 & 1.1950 & 1.1070 & 124.18 \\
\hline 3 & $2{ }^{3} B_{2}$ & $n_{\mathrm{N}\left(2 p_{x}\right)} \rightarrow r_{\mathrm{C}\left(3 p_{y}\right)}$ & 85.1 & 82.7 & 1.1963 & 1.1210 & 127.21 \\
\hline 4 & $1^{3} A_{1}$ & $n_{\mathrm{N}\left(2 p_{x}\right)} / \sigma_{\mathrm{CH}}(2) \rightarrow \pi_{\mathrm{CN}}^{*}$ & 92.3 & 88.1 & 1.1897 & 1.1078 & 122.16 \\
\hline 5 & $2^{3} A_{1}$ & $\pi_{\mathrm{CN}} \rightarrow \pi_{\mathrm{CN}}^{*}$ & 100.2 & 77.1 & 1.4358 & 1.0981 & 124.04 \\
\hline 6 & $2^{3} A_{2}$ & $n_{\mathrm{N}\left(2 p_{x}\right)} \rightarrow r_{\mathrm{C}\left(3 p_{z}\right)} / \pi_{\mathrm{CN}}^{*} / r_{\mathrm{N}\left(3 p_{z}\right)}$ & 112.1 & 110.6 & 1.2134 & 1.1019 & 123.56 \\
\hline 7 & $1^{3} B_{1}$ & $\pi_{\mathrm{CN}} \rightarrow r_{\mathrm{C}(3 s)}$ & 135.6 & 123.9 & 1.3722 & 1.0794 & 120.90 \\
\hline 8 & $2^{3} B_{1}$ & $n_{\mathrm{N}\left(2 p_{\vartheta}\right)} \rightarrow \pi_{\mathrm{CN}}^{*}$ & 144.5 & 128.0 & 1.3922 & 1.0755 & 119.92 \\
\hline 11 & $3^{3} B_{1}$ & $\pi_{\mathrm{CN}} \rightarrow r_{\mathrm{C}\left(3 p_{y}\right)} / r_{\mathrm{N}(3 s)}$ & 153.7 & 141.6 & 1.3697 & 1.0818 & 122.93 \\
\hline 13 & $3^{3} A_{2}$ & $\pi_{\mathrm{CN}} \rightarrow r_{\mathrm{C}\left(3 p_{x}\right)} / \pi_{\mathrm{CN}}^{*}$ & 158.0 & 146.7 & 1.3553 & 1.0778 & 120.27 \\
\hline
\end{tabular}

${ }^{\mathrm{a}} \phi_{\mathrm{HCNH}}=53.95^{\circ}$.

TABLE VI. Vertical excitation energies ( $\mathrm{kcal} / \mathrm{mol})$, natural charge shifts, adiabatic excitation energies, and optimized geometries for $\mathrm{CH}_{2} \mathrm{OH}^{+}$.

\begin{tabular}{|c|c|c|c|c|c|c|c|c|c|}
\hline State & Symmetry & Transition & VEE & $\Delta q_{\mathrm{C}}$ & $\Delta q_{\mathrm{o}}$ & AEE & $r_{\mathrm{CO}}$ & $r_{\mathrm{OH}_{5}}$ & $\theta_{\mathrm{COH}_{5}}$ \\
\hline \multicolumn{10}{|c|}{ Ground state } \\
\hline 0 & $2{ }^{1} A^{\prime \prime}$ & & & & & & 1.2317 & 0.9600 & 117.75 \\
\hline \multicolumn{10}{|c|}{ Singlet states } \\
\hline $1^{\mathrm{a}}$ & $1{ }^{1} A^{\prime \prime}$ & $\pi_{\mathrm{CO}} / \sigma_{\mathrm{CH}}(2) / \sigma_{\mathrm{OH}} \rightarrow \pi_{\mathrm{CO}}^{*}$ & 164.6 & -0.525 & +0.154 & 131.5 & 1.2540 & 0.9685 & 119.09 \\
\hline 2 & $2{ }^{1} A^{\prime \prime}$ & $\sigma_{\mathrm{CO}} / \sigma_{\mathrm{CH}_{3}} / \pi_{\mathrm{CO}} \rightarrow \sigma_{\mathrm{CO}}^{*} / n_{\mathrm{C}\left(2 p_{v x}\right)}$ & 256.0 & -0.430 & +0.257 & \multicolumn{4}{|c|}{ Unresolvable curve crossing } \\
\hline $3^{\mathrm{a}}$ & $2{ }^{1} A^{\prime}$ & $\pi_{\mathrm{CO}} \rightarrow \pi_{\mathrm{CO}}^{*}$ & 260.8 & -0.383 & +0.408 & 131.5 & 1.2540 & 0.9685 & 119.09 \\
\hline 4 & $3{ }^{1} A^{\prime}$ & $n_{\mathrm{O}} / \sigma_{\mathrm{CH}}(2) \rightarrow \sigma_{\mathrm{OH}}^{*} / r_{\mathrm{C}(3 s p)} / r_{\mathrm{O}(3 s)}$ & 297.1 & -0.130 & +0.240 & \multicolumn{4}{|c|}{ Dissociative- $\mathrm{O}-\mathrm{H}$ bond broken } \\
\hline $5^{\mathrm{a}}$ & $3{ }^{1} A^{\prime \prime}$ & $\pi_{\mathrm{CO}} / \sigma_{\mathrm{OH}} / \sigma_{\mathrm{CH}_{4}} \rightarrow \pi_{\mathrm{CO}}^{*}$ & 312.8 & -0.420 & +0.266 & 131.5 & 1.2540 & 0.9685 & 119.09 \\
\hline 6 & $4{ }^{1} A^{\prime \prime}$ & $\pi_{\mathrm{CO}} \rightarrow \sigma_{\mathrm{OH}}^{*} / \pi_{\mathrm{CO}}^{*} / r_{\mathrm{H}_{5}}$ & 321.8 & -0.040 & +0.503 & \multicolumn{4}{|c|}{ Dissociative- $\mathrm{O}-\mathrm{H}$ bond broken } \\
\hline 7 & $4{ }^{1} A^{\prime}$ & $\pi_{\mathrm{CO}} / \sigma_{\mathrm{CH}}(2) / \sigma_{\mathrm{OH}} / n_{\mathrm{O}} \rightarrow \pi_{\mathrm{CO}}^{*} / r_{\mathrm{C}\left(3 s p_{v}\right)} / \sigma_{\mathrm{CH}}$ & 336.6 & -0.186 & +0.152 & \multicolumn{4}{|c|}{ Dissociative $-\mathrm{C}-\mathrm{H}$ bonds broken } \\
\hline $8^{\mathrm{a}}$ & $5^{1} A^{\prime \prime}$ & $\sigma_{\mathrm{CH}_{4}} / n_{\mathrm{O}} \rightarrow r_{\mathrm{C}\left(3 p_{x y}\right)} / \pi_{\mathrm{CO}}^{*} / r_{\mathrm{O}\left(3 s p_{x}\right)}$ & 353.2 & -0.354 & +0.156 & 131.5 & 1.2540 & 0.9685 & 119.09 \\
\hline \multicolumn{10}{|c|}{ Triplet states } \\
\hline $1^{\mathrm{b}}$ & $1^{3} A^{\prime \prime}$ & $\pi_{\mathrm{CO}} / \sigma_{\mathrm{CH}_{4}} / \sigma_{\mathrm{CH}_{3}} / \sigma_{\mathrm{OH}} \rightarrow \pi_{\mathrm{CO}}^{*}$ & 141.8 & -0.524 & +0.126 & 108.3 & 1.2538 & 0.9631 & 118.59 \\
\hline $2^{\mathrm{b}}$ & $1^{3} A^{\prime}$ & $\pi_{\mathrm{CO}} \rightarrow \pi_{\mathrm{CO}}^{*}$ & 161.4 & -0.738 & +0.764 & 108.3 & 1.2538 & 0.9631 & 118.59 \\
\hline 3 & $2^{3} A^{\prime \prime}$ & $\sigma_{\mathrm{CO}} / \sigma_{\mathrm{CH}_{3}} \rightarrow \sigma_{\mathrm{CO}}^{*} / \sigma_{\mathrm{CH}_{3}}^{*}$ & 227.7 & -0.432 & +0.202 & \multicolumn{4}{|c|}{ Dissociative $-\mathrm{C}-\mathrm{H}_{3}$ bond broken } \\
\hline 4 & $2^{3} A^{\prime}$ & $\sigma_{\mathrm{OH}} / \sigma_{\mathrm{CH}_{4}} / n_{\mathrm{O}} / \sigma_{\mathrm{CH}_{3}} \rightarrow \sigma_{\mathrm{OH}^{\prime}}^{*} / \sigma_{\mathrm{CH}_{4}}^{*}$ & 274.3 & -0.055 & +0.303 & \multicolumn{4}{|c|}{ Dissociative $-\mathrm{O}-\mathrm{H}$ bond broken } \\
\hline 5 & $3^{3} A^{\prime \prime}$ & $\pi_{\mathrm{CO}} / \sigma_{\mathrm{CH}_{4}} / \sigma_{\mathrm{OH}} \rightarrow \pi_{\mathrm{CO}}^{*} / \sigma_{\mathrm{CH}_{4}}^{*}$ & 279.6 & -0.414 & +0.188 & \multicolumn{4}{|c|}{ Dissociative $-\mathrm{C}-\mathrm{H}_{4}$ bond broken } \\
\hline 6 & $4^{3} A^{\prime \prime}$ & $\pi_{\mathrm{CO}} \rightarrow \sigma_{\mathrm{OH}}^{*} / r_{\mathrm{H}_{5}(2 s)}$ & 303.1 & +0.102 & +0.115 & \multicolumn{4}{|c|}{ Dissociative- $\mathrm{O}-\mathrm{H}$ bond broken } \\
\hline 7 & $3^{3} A^{\prime}$ & $\sigma_{\mathrm{CH}}(2) / \sigma_{\mathrm{OH}} / n_{\mathrm{O}} \rightarrow \sigma_{\mathrm{CH}}^{*} / \sigma_{\mathrm{OH}}^{*} / r_{\mathrm{H}_{3}(2 s)}$ & 305.0 & -0.009 & +0.537 & \multicolumn{4}{|c|}{ Dissociative- $\mathrm{O}-\mathrm{H}$ bond broken } \\
\hline 8 & $4^{3} A^{\prime}$ & $\sigma_{\mathrm{CH}}(2) / n_{\mathrm{O}} / \sigma_{\mathrm{OH}} \rightarrow \sigma_{\mathrm{CH}_{4}}^{*} / r_{\mathrm{C}\left(3 s p_{x y}\right)} / \sigma_{\mathrm{CH}_{3}}^{*}$ & 320.9 & -0.008 & +0.047 & \multicolumn{4}{|c|}{ Dissociative $-\mathrm{C}-\mathrm{H}_{4}$ bond broken } \\
\hline
\end{tabular}

${ }^{\mathrm{a}} \phi_{\mathrm{H}_{4} \mathrm{COH}_{3}}=52.06^{\circ}$ (pyramidal distortion angle), $\mathrm{H}_{5}$ opposite $\left(180^{\circ}\right.$ from) bisector of $\mathrm{HCH}$ angle.

${ }^{\mathrm{b}} \phi_{\mathrm{H}_{4} \mathrm{OCH}_{3}}=75.02^{\circ}, \mathrm{H}_{5}$ opposite bisector of $\mathrm{HCH}$ angle. 
TABLE VII. Vertical excitation energies (kcal/mol), adiabatic excitation energies, and optimized geometries for formaldehyde.

\begin{tabular}{|c|c|c|c|c|c|c|c|c|c|}
\hline State & Symmetry & Transition & $\mathrm{VEE}^{\mathrm{a}}$ & Hadad $^{\mathrm{b}}$ & AEE & $r_{\mathrm{CO}}$ & $r_{\mathrm{CH}}$ & $\theta_{\mathrm{OCH}}$ & $\phi_{\mathrm{HCOH}}$ \\
\hline \multicolumn{10}{|c|}{ Ground state } \\
\hline 0 & $2{ }^{1} A_{1}$ & & & & & 1.1862 & 1.0923 & 121.83 & 0.00 \\
\hline \multicolumn{10}{|c|}{ Singlet states } \\
\hline 1 & $1{ }^{1} A_{2}$ & $n_{\mathrm{O}\left(2 p_{x}\right)} \rightarrow \pi_{\mathrm{CO}}^{*}$ & 108.2 & 105.6 & 103.5 & 1.2552 & 1.0856 & 118.01 & 26.10 \\
\hline 2 & $1^{1} B_{2}$ & $n_{\mathrm{O}\left(2 p_{x}\right)} / \sigma_{\mathrm{CH}}(2) \rightarrow r_{\mathrm{C}(3 s)} / \sigma_{\mathrm{CH}}^{*}(2)$ & 203.3 & 158.0 & 197.8 & 1.1311 & 1.1842 & 130.73 & 0.00 \\
\hline 3 & $2{ }^{1} B_{2}$ & $n_{\mathrm{O}\left(2 p_{x}\right.} / \sigma_{\mathrm{CH}}(2) \rightarrow r_{\mathrm{C}\left(3 p_{y}\right)} / \sigma_{\mathrm{CO}}^{*}$ & 219.3 & 176.6 & 214.5 & 1.1441 & 1.1452 & 127.86 & 0.00 \\
\hline 4 & $2{ }^{1} A_{1}$ & $n_{\mathrm{O}\left(2 p_{x}\right)} / \sigma_{\mathrm{CH}}(2) \rightarrow r_{\mathrm{C}\left(3 p_{x}\right)}$ & 227.5 & 195.3 & 186.4 & 1.4889 & 1.0774 & 107.97 & 44.72 \\
\hline 5 & $1{ }^{1} B_{1}$ & $\pi_{\mathrm{CO}} / n_{\mathrm{O}\left(2 s p_{y}\right)} \rightarrow \pi_{\mathrm{CO}}^{*} / \sigma_{\mathrm{CO}}^{*}$ & 234.2 & 229.9 & 186.4 & 1.4889 & 1.0774 & 107.97 & 44.72 \\
\hline 6 & $3{ }^{1} A_{1}$ & $\pi_{\mathrm{CO}} / n_{\mathrm{O}\left(2 s p_{y}\right)} \rightarrow \pi_{\mathrm{CO}}^{*}$ & 235.4 & 211.9 & 186.4 & 1.4889 & 1.0774 & 107.97 & 44.72 \\
\hline 8 & $3{ }^{1} A_{2}$ & $\sigma_{\mathrm{CH}}(2) / n_{\mathrm{O}\left(2 p_{x}\right)} \rightarrow \pi_{\mathrm{CO}}^{*}$ & 269.9 & 232.4 & 214.5 & 1.1441 & 1.1452 & 127.86 & 0.00 \\
\hline 9 & $2{ }^{1} B_{1}$ & $\pi_{\mathrm{CO}} \rightarrow r_{\mathrm{C}(3 s)} / r_{\mathrm{O}(3 s)}$ & 270.0 & 250.0 & 254.4 & 1.3131 & 1.0767 & 116.98 & 0.00 \\
\hline \multicolumn{10}{|c|}{ Triplet states } \\
\hline 1 & $1^{3} A_{2}$ & $n_{\mathrm{O}\left(2 p_{x}\right)} \rightarrow \pi_{\mathrm{CO}}^{*}$ & 89.8 & 95.7 & 82.8 & 1.2552 & 1.0926 & 114.26 & 47.42 \\
\hline 2 & $1^{3} A_{1}$ & $\pi_{\mathrm{CO}} \rightarrow \pi_{\mathrm{CO}}^{*}$ & 120.9 & 155.0 & 82.5 & 1.4080 & 1.0726 & 113.24 & 40.00 \\
\hline 3 & $1^{3} B_{2}$ & $n_{\mathrm{O}\left(2 p_{x}\right.} / \sigma_{\mathrm{CH}}(2) \rightarrow r_{\mathrm{C}(3 s)} / \sigma_{\mathrm{CH}}^{*}(2)$ & 193.8 & 160.7 & 82.8 & 1.2552 & 1.0926 & 114.26 & 47.42 \\
\hline 4 & $1^{3} B_{1}$ & $n_{\mathrm{O}\left(2 s p_{y}\right)} \rightarrow \pi_{\mathrm{CO}}^{*}$ & 203.9 & 211.7 & 164.3 & 1.4681 & 1.0731 & 105.89 & 37.01 \\
\hline 5 & $2^{3} B_{2}$ & $n_{\mathrm{O}\left(2 p_{y}\right)} / \sigma_{\mathrm{CH}}(2) \rightarrow r_{\mathrm{C}\left(3 p_{y}\right)} / r_{\mathrm{O}(3 s)} / \sigma_{\mathrm{CO}}^{*}$ & 211.0 & 178.7 & 206.9 & 1.1504 & 1.1368 & 127.55 & 0.00 \\
\hline 6 & $2^{3} A_{1}$ & $n_{\mathrm{O}\left(2 p_{x}\right)} / \sigma_{\mathrm{CH}}(2) \rightarrow r_{\mathrm{C}\left(3 p_{x}\right)}$ & 218.6 & 179.4 & 211.3 & 1.1385 & 1.2027 & 124.02 & 0.00 \\
\hline 8 & $3^{3} A_{2}$ & $\sigma_{\mathrm{CH}}(2) / n_{\mathrm{O}\left(2 p_{x}\right)} \rightarrow \pi_{\mathrm{CO}}^{*}$ & 249.2 & 242.6 & 218.1 & 1.2926 & 1.1601 & 132.00 & 28.56 \\
\hline 10 & $2{ }^{3} \mathrm{~B}_{2}$ & $n_{\mathrm{O}\left(2 p_{x}\right)} / \sigma_{\mathrm{CH}}(2) \rightarrow \pi_{\mathrm{CO}}^{*} / r_{\mathrm{C}\left(3 s p_{y}\right)} / \sigma_{\mathrm{CH}}^{*}(2)$ & 273.1 & & 237.8 & 1.1444 & 1.2144 & 111.39 & 0.00 \\
\hline
\end{tabular}

${ }^{\text {a This work. }}$

${ }^{\mathrm{b}}$ Reference 10, CIS-MP2/6-311(2+,2+)G**.

TABLE VIII. Vertical excitation energies ( $\mathrm{kcal} / \mathrm{mol})$, adiabatic excitation energies, and optimized geometries for $\mathrm{HCO}^{-}$.

\begin{tabular}{|c|c|c|c|c|c|c|c|}
\hline State & Symmetry & Transition & VEE & AEE & $r_{\mathrm{CO}}$ & $r_{\mathrm{CH}}$ & $\theta_{\mathrm{HCO}}$ \\
\hline \multicolumn{8}{|c|}{ Ground state } \\
\hline 0 & $2{ }^{1} A^{\prime}$ & & & & 1.2241 & 1.1895 & 109.33 \\
\hline \multicolumn{8}{|c|}{ Singlet states } \\
\hline 1 & $1{ }^{1} A^{\prime \prime}$ & $n_{\mathrm{C}\left(2 s p_{x}\right)} / n_{\mathrm{O}\left(2 p_{x z}\right.} \rightarrow \pi_{\mathrm{CO}}^{*} / \sigma_{\mathrm{CH}}^{*} / r_{\mathrm{C}\left(3 p_{z}\right)}$ & 53.3 & 47.4 & 1.2175 & 1.1102 & 128.49 \\
\hline 2 & $2{ }^{1} A^{\prime}$ & $n_{\mathrm{C}\left(2 s p_{x y}\right)} / n_{\mathrm{O}\left(2 p_{x}\right)} \rightarrow r_{\mathrm{C}\left(3 p_{x}\right)} / \sigma_{\mathrm{CH}}^{*}$ & 71.7 & 60.8 & 1.1590 & 1.0983 & 125.76 \\
\hline 3 & $3{ }^{1} A^{\prime}$ & $n_{\mathrm{C}\left(2 s p_{x y}\right)} / n_{\mathrm{O}\left(2 p_{x}\right)} \rightarrow \sigma_{\mathrm{CO}}^{*} / \sigma_{\mathrm{CH}}^{*}$ & 84.6 & 73.7 & 1.1590 & 1.1300 & 128.16 \\
\hline 4 & $2{ }^{1} A^{\prime \prime}$ & $n_{\mathrm{C}\left(2 s p_{x y}\right)} / n_{\mathrm{O}\left(2 p_{x}\right)} \rightarrow r_{\mathrm{C}\left(3 p_{z}\right)} / \sigma_{\mathrm{CO}}^{*}$ & 107.9 & 99.9 & 1.1864 & 1.1078 & 127.87 \\
\hline 5 & $4{ }^{1} A^{\prime}$ & $n_{\mathrm{C}\left(2 s p_{x y}\right)} / n_{\mathrm{O}\left(2 p_{x}\right)} / \sigma_{\mathrm{CH}} \rightarrow r_{\mathrm{C}\left(3 p_{x}\right)}$ & 110.3 & 105.8 & 1.1711 & 1.1666 & 117.44 \\
\hline 6 & $3{ }^{1} A^{\prime \prime}$ & $n_{\mathrm{C}\left(2 s p_{x y}\right)} / n_{\mathrm{O}\left(2 p_{x}\right)} \rightarrow \pi_{\mathrm{CO}}^{*} / r_{\mathrm{C}\left(3 p_{z}\right)}$ & 182.5 & 159.2 & 1.4113 & 1.1230 & 102.94 \\
\hline 7 & $5^{1} A^{\prime}$ & $\left.n_{\mathrm{O}\left(2 p_{x} x\right.}\right) / n_{\mathrm{C}\left(2 s p_{x y}\right)} / \sigma_{\mathrm{CH}} \rightarrow r_{\mathrm{O}\left(3 p_{y}\right)} / r_{\mathrm{C}\left(3 s p_{y}\right)}$ & 187.6 & 181.3 & 1.1554 & 1.1315 & 122.37 \\
\hline 8 & $6{ }^{1} A^{\prime}$ & $\pi_{\mathrm{CO}} / n_{\mathrm{O}\left(2 p_{x}\right)} / n_{\mathrm{C}\left(2 s p_{x y}\right)} / \sigma_{\mathrm{CH}} \rightarrow r_{\mathrm{C}\left(3 p_{x}\right)} / \pi_{\mathrm{CO}}^{*} / r_{\mathrm{C}\left(3 p_{z}\right)}$ & 202.9 & 180.2 & 1.4716 & 1.1104 & 102.42 \\
\hline 9 & $4{ }^{1} A^{\prime \prime}$ & $\pi_{\mathrm{CO}} \rightarrow r_{\mathrm{C}\left(3 s p_{y}\right)} / r_{\mathrm{O}(3 s)}$ & 206.5 & 187.8 & 1.3770 & 1.0931 & 103.47 \\
\hline 10 & $7{ }^{1} A^{\prime}$ & $\pi_{\mathrm{CO}} / n_{\mathrm{C}\left(2 p_{x y}\right)} / \sigma_{\mathrm{CH}} \rightarrow \pi_{\mathrm{CO}}^{*} / r_{\mathrm{O}\left(3 p_{z}\right)} / r_{\mathrm{C}\left(3 p_{z}\right)}$ & 210.7 & 210.2 & 1.2124 & 1.1571 & 112.55 \\
\hline 15 & $6{ }^{1} A^{\prime \prime}$ & $\sigma_{\mathrm{CO}} / \sigma_{\mathrm{CH}} / \pi_{\mathrm{CO}} / n_{\mathrm{O}\left(2 p_{x}\right)} \rightarrow \pi_{\mathrm{CO}}^{*}$ & 238.9 & 197.8 & 1.4747 & 1.1524 & 99.95 \\
\hline \multicolumn{8}{|c|}{ Triplet states } \\
\hline 1 & $1^{3} A^{\prime \prime}$ & $n_{\mathrm{C}\left(2 s p_{x z}\right)} / n_{\mathrm{O}\left(2 p_{x z}\right)} \rightarrow \pi_{\mathrm{CO}}^{*} / \sigma_{\mathrm{CO}}^{*}$ & 27.6 & 20.6 & 1.2461 & 1.1044 & 125.86 \\
\hline 2 & $1{ }^{3} A^{\prime}$ & $n_{\mathrm{C}\left(2 s p_{x y}\right)} / n_{\mathrm{O}\left(2 p_{x}\right)} \rightarrow r_{\mathrm{C}(3 s)} / \sigma_{\mathrm{CH}}^{*}$ & 60.6 & 48.8 & 1.1554 & 1.1144 & 128.08 \\
\hline 3 & $2{ }^{3} A^{\prime}$ & $n_{\mathrm{C}\left(2 s p_{x z}\right)} / n_{\mathrm{O}\left(2 p_{x z}\right)} \rightarrow r_{\mathrm{C}\left(3 p_{x y}\right)} / \sigma_{\mathrm{CH}}^{*}$ & 76.3 & 63.1 & 1.1491 & 1.1187 & 129.97 \\
\hline 4 & $3^{3} A^{\prime}$ & $\left.n_{\mathrm{C}\left(2 s p_{x y}\right.}\right) / n_{\mathrm{O}\left(2 p_{x}\right)} \rightarrow r_{\mathrm{C}\left(3 p_{x y}\right)} / r_{\mathrm{O}(3 s)} / \sigma_{\mathrm{CH}}^{*}$ & 80.1 & 68.3 & 1.1556 & 1.1249 & 129.25 \\
\hline 5 & $2^{3} A^{\prime \prime}$ & $n_{\mathrm{C}\left(2 s p_{x z}\right.} / n_{\mathrm{O}\left(2 p_{x z}\right)} \rightarrow \sigma_{\mathrm{CH}}^{*} / \pi_{\mathrm{CO}}^{*}$ & 96.3 & 87.8 & 1.1760 & 1.1184 & 126.33 \\
\hline 6 & $4^{3} A^{\prime}$ & $\pi_{\mathrm{CO}} \rightarrow \pi_{\mathrm{CO}}^{*}$ & 134.9 & 102.1 & 1.4422 & 1.1149 & 101.87 \\
\hline 7 & $3^{3} A^{\prime \prime}$ & $n_{\mathrm{C}\left(2 s p_{x y}\right)} / n_{\mathrm{O}\left(2 p_{x}\right)} \rightarrow \pi_{\mathrm{CO}}^{*}$ & 158.4 & 141.3 & 1.3454 & 1.1268 & 101.55 \\
\hline 8 & $5^{3} A^{\prime}$ & $n_{\mathrm{C}\left(2 s p_{x y}\right)} / n_{\mathrm{O}\left(2 p_{x}\right)} \rightarrow r_{\mathrm{C}\left(3 s p_{x y}\right)} / r_{\mathrm{O}\left(3 p_{y}\right)} / \sigma_{\mathrm{CH}}^{*}$ & 163.9 & \multicolumn{4}{|c|}{ Dissociative $-\mathrm{C}-\mathrm{H}$ bond broken } \\
\hline 9 & $6{ }^{3} A^{\prime}$ & $n_{\mathrm{C}\left(2 s p_{x y}\right)} / n_{\mathrm{O}\left(2 p_{x}\right)} / \sigma_{\mathrm{CH}} \rightarrow r_{\mathrm{O}\left(3 p_{y}\right)} / \sigma_{\mathrm{CH}}^{*} / r_{\mathrm{C}\left(3 s p_{x y}\right)}$ & 191.2 & \multicolumn{4}{|c|}{ Dissociative $-\mathrm{C}-\mathrm{H}$ bond broken } \\
\hline 10 & $7{ }^{3} A^{\prime}$ & $n_{\mathrm{O}\left(2 p_{x}\right)} / n_{\mathrm{C}\left(2 s p_{x y}\right)} / \sigma_{\mathrm{CH}} \rightarrow r_{\mathrm{C}\left(3 p_{x}\right)} / r_{\mathrm{O}\left(3 p_{x}\right)}$ & 197.5 & 191.6 & 1.1566 & 1.1376 & 115.68 \\
\hline 11 & $4^{3} A^{\prime \prime}$ & $\pi_{\mathrm{CO}} / \sigma_{\mathrm{CH}} \rightarrow \pi_{\mathrm{CO}}^{*} / r_{\mathrm{C}\left(3 s p_{y}\right)} / r_{\mathrm{O}(3 s)}$ & 199.5 & 181.2 & 1.3837 & 1.0948 & 103.24 \\
\hline 14 & $6^{3} A^{\prime \prime}$ & $n_{\mathrm{O}\left(2 p_{x y}\right)} / n_{\mathrm{C}\left(2 s p_{x y}\right)} / \sigma_{\mathrm{CH}} \rightarrow \pi_{\mathrm{CO}}^{*} / r_{\mathrm{O}\left(3 p_{z}\right)}$ & 224.5 & 221.9 & 1.2563 & 1.1364 & 112.23 \\
\hline
\end{tabular}




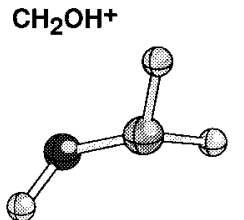

a) singlet $1\left({ }^{1} A^{\prime \prime}\right)$

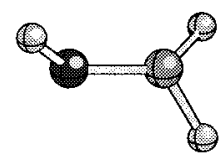

b) triplet $1\left({ }^{3} A^{\prime \prime}\right)$

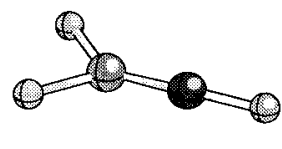

c) triplet $9\left({ }^{3} A^{\prime}\right)$

FIG. 4. Nonplanar excited state optimized geometries (CIS 6-31+ $\mathrm{G}^{*}$ ) of $\mathrm{CH}_{2} \mathrm{OH}^{+}$.

\section{Formaldehyde}

The excited states of protonated formaldehyde, $\mathrm{CH}_{2} \mathrm{OH}^{+}$, are listed in Table VI. Over the series $\mathrm{CH}_{2} \mathrm{CH}^{-}$, $\mathrm{CH}_{2} \mathrm{NH}, \mathrm{CH}_{2} \mathrm{OH}^{+}$, as the electronegativity difference between $\mathrm{C}$ and its partner atom increases, and the overall charge of the species becomes more positive, the states become more widely separated. The greater shift in charge toward the carbon atom upon excitation in the molecules with more polar CX bonds is due to two factors. The first is the transfer of more density from orbitals centered on $\mathrm{X}_{2}\left(n_{\mathrm{X}_{1}}\right)$ to Rydbergs on $\mathrm{C}_{1}$ as the $\mathrm{X}$ atom becomes more

electronegative. ${ }^{51}$ The second factor is the increased polarization of the $\pi$ bond toward $\mathrm{X}_{2}$ and $\pi^{*}$ toward $\mathrm{C}_{1}$ as $\mathrm{X}_{2}$ becomes more electronegative. ${ }^{52}$

Table VII lists the excited state assignments and vertical transition energies of $\mathrm{CH}_{2} \mathrm{O}$, along with the CIS-MP2/6-311(2+,2+) $\mathrm{G}^{* *}$ results of Hadad et al. ${ }^{14}$ Most of the assignments and valence state energies agree fairly well, but their CIS-MP2 Rydberg excitation energies are lower due to the effect of electron correlation. Two lone pairs on the same atom remain distinct from each other, hybridized $2 p_{x}$ and $2 s p_{y}$. However, when two lone pairs are on adjacent heavy atoms, as in $\mathrm{HCO}^{-}$(Table VIII), the excitations become noticeably more delocalized. It also takes much less energy to excite an electron out of the HOMO, which is a combination of the $\mathrm{C}$ and $\mathrm{O}$ lone pairs (more out of the less stable $n_{\mathrm{C}}$ lone pair than $n_{\mathrm{O}}$ ). Following the series $\mathrm{CH}_{2} \mathrm{CH}^{-}, \mathrm{CH}_{2} \mathrm{~N}^{-}, \mathrm{HCO}^{-}$, one sees the first singlet and triplet energies diminish as the species maintains a negative charge but hydrogens are lost.

Table VI shows even more curve crossing and dissociation for excited $\mathrm{CH}_{2} \mathrm{OH}^{+}$than for $\mathrm{CH}_{2} \mathrm{NH}_{2}^{+}$. The shift in density away from the oxygen toward carbon upon excitation into the $\sigma_{\mathrm{OH}}^{*}$ antibond weakens the $\mathrm{OH}$ bond. The most

TABLE IX. Excited state deprotonation energies $(\mathrm{kcal} / \mathrm{mol})$ for $\mathrm{CH}_{2} \mathrm{CH}_{2}$.

\begin{tabular}{|c|c|c|c|}
\hline Deprotonation reaction $^{\mathrm{a}}$ & Transition $^{\mathrm{b}}$ & $\begin{array}{l}\text { Vertical } \\
\text { deprotonation } \\
\text { energy }^{c}\end{array}$ & $\begin{array}{c}\text { Adiabatic } \\
\text { deprotonation } \\
\text { energy }\end{array}$ \\
\hline $\begin{array}{l}\text { Ground state } \\
\mathrm{CH}_{2} \mathrm{CH}_{2}(0) \rightarrow \mathrm{CH}_{2} \mathrm{CH}^{-}(0)+\mathrm{H}^{+}\end{array}$ & $\cdots$ & 422.6 & 422.6 \\
\hline Singlet states & & & \\
\hline 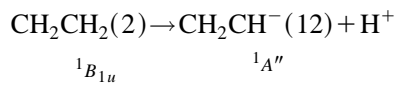 & $\pi_{\mathrm{CC}} /\left[n_{\mathrm{C}}\right] \rightarrow \pi_{\mathrm{CC}}^{*} / r_{\mathrm{C}\left(3 p_{z}\right)}$ & 401.4 & 452.5 \\
\hline $\begin{array}{c}\mathrm{CH}_{2} \mathrm{CH}_{2}(3) \\
{ }^{1} B_{1 g}\end{array} \underset{\mathrm{CH}_{2} \mathrm{CH}^{-}(7)+\mathrm{H}^{+}}{{ }^{1} A^{\prime}}$ & $\pi_{\mathrm{CC}} \rightarrow r_{\mathrm{C}\left(3 p_{x}\right)}$ & 364.3 & 340.9 \\
\hline $\begin{array}{c}\mathrm{CH}_{2} \mathrm{CH}_{2}(4) \rightarrow{ }^{1} B_{2 g} \\
\mathrm{CH}_{2} \mathrm{CH}^{-}(13)+\mathrm{H}^{+} \\
{ }^{1} A^{\prime \prime}\end{array}$ & $\pi_{\mathrm{CC}} \rightarrow r_{\mathrm{C}\left(3 p_{y}\right)}$ & 396.2 & 398.0 \\
\hline $\begin{array}{c}\mathrm{CH}_{2} \mathrm{CH}_{2}(5) \\
{ }^{1} A_{g}\end{array} \underset{{ }^{1} A^{\prime}}{\mathrm{CH}_{2} \mathrm{CH}^{-}(11)+\mathrm{H}^{+}}$ & $\pi_{\mathrm{CC}} /\left[n_{\mathrm{CC}}\right] \rightarrow r_{\mathrm{C}\left(3 p_{z}\right)} / \pi_{\mathrm{CC}}^{*}$ & 363.4 & 366.7 \\
\hline $\begin{array}{l}\mathrm{CH}_{2} \mathrm{CH}_{2}(9) \rightarrow \mathrm{CH}_{2} \mathrm{CH}^{-}(17)+\mathrm{H}^{+} \\
\stackrel{{ }^{1} B_{2 g}}{{ }^{1} A^{\prime \prime}} \\
\text { Triplet states }\end{array}$ & $\sigma_{\mathrm{CC}} / \sigma_{\mathrm{CH}} \rightarrow \pi_{\mathrm{CC}}^{*}$ & 391.8 & 292.1 \\
\hline $\begin{array}{c}\mathrm{CH}_{2} \mathrm{CH}_{2}(1) \\
{ }^{3} B_{1 u}\end{array} \underset{\mathrm{CH}_{2} \mathrm{CH}^{-}(3)+\mathrm{H}^{+}}{{ }^{3} A^{\prime \prime}}$ & $\pi_{\mathrm{CC}} \rightarrow \pi_{\mathrm{CC}}^{*}$ & 425.4 & 414.1 \\
\hline $\begin{array}{c}\mathrm{CH}_{2} \mathrm{CH}_{2}(3) \\
{ }^{3} B_{1 g}\end{array} \underset{{ }^{3} A^{\prime}}{\mathrm{CH}_{2} \mathrm{CH}^{-}(9)}+\mathrm{H}^{+}$ & $\pi_{\mathrm{CC}} \rightarrow r_{\mathrm{C}\left(3 p_{x}\right)}$ & 363.4 & 338.7 \\
\hline $\begin{array}{c}\mathrm{CH}_{2} \mathrm{CH}_{2}(4) \rightarrow \mathrm{CH}_{2} \mathrm{CH}^{-}(6)+\mathrm{H}^{+} \\
{ }^{3} B_{2 g}\end{array}$ & $\pi_{\mathrm{CC}} \rightarrow r_{\mathrm{C}\left(3 p_{y}\right)}$ & 342.5 & 326.1 \\
\hline$\underset{\mathrm{CH}_{2} \mathrm{CH}_{2}(5)}{{ }^{3} A_{g}} \rightarrow \underset{{ }^{3} A^{\prime}}{\mathrm{CH}_{2} \mathrm{CH}^{-}(8)}+\mathrm{H}^{+}$ & $\pi_{\mathrm{CC}} \rightarrow r_{\mathrm{C}\left(3 p_{z}\right)} / \pi_{\mathrm{CC}}^{*}$ & 358.1 & 358.9 \\
\hline$\underset{{ }^{3} \mathrm{CH}_{2 g} \mathrm{CH}_{2}(9)}{\mathrm{CH}_{2} \mathrm{CH}^{-}(18)+\mathrm{H}^{+}}{ }_{{ }^{3} A^{\prime \prime}}$ & $\sigma_{\mathrm{CC}} / \sigma_{\mathrm{CH}} \rightarrow \pi_{\mathrm{CC}}^{*}$ & 393.9 & 337.5 \\
\hline
\end{tabular}

${ }^{\mathrm{a}}$ Numbers in parentheses refer to states numbered in earlier tables.

brbitals in brackets make a minor contribution to the excitation in one species, and none in the other.

${ }^{c}$ Difference in vertical excitation energies (no geometric relaxation). 
stable excited state geometries have the $\mathrm{OH}$ bond $180^{\circ}$ opposite the bisector of the $\mathrm{HCH}$ angle [Figs. 4(a) and 4(b)]. The ninth triplet state relieves strain by linearizing the $\mathrm{COH}$ angle.

The correlation of $\pi / \pi^{*}$ character and $\mathrm{CO}$ bond lengthening and nonplanarity for the excited states of $\mathrm{CH}_{2} \mathrm{O}$ can be seen in Table VII. The CO bond length increases by $0.3 \AA$ for the formaldehyde adiabatic $\pi \rightarrow \pi^{*}$ singlet state, the $\mathrm{HCH}$ angle opens up by $27^{\circ}$, and the geometry distorts to a nonplanar structure. This contrasts with the structure found by Hadad et al., ${ }^{14}$ which had similar bond lengths and angles, but was planar. The $\pi \rightarrow \pi^{*}$ triplet state CO bond lengthens by a lesser amount. The CO bond lengths for $n_{\mathrm{O}} \rightarrow \pi^{*}$ states increase slightly, but the structure is even more pyramidal.

The optimized geometries of $\mathrm{CH}_{2} \mathrm{O}$ states exciting out of MO 8 (the HOMO) composed of $n_{\mathrm{O}\left(2 p_{x}\right)} / \sigma_{\mathrm{CH}}(2)$ have shorter CO bond lengths. Depopulation of the oxygen lone pair decreases the diffuseness of the density around oxygen and moves electrons toward the $\mathrm{CO} \sigma$ bond to strengthen it, confirmed by contour plots of these orbitals. These results are consistent with the CIS optimized structures calculated by Hadad et al. ${ }^{14}$ for the states labeled $n_{y} \rightarrow 3 s\left(1{ }^{1} B_{2}\right)$ and $n_{y} \rightarrow 3 p_{z}\left(2{ }^{1} B_{2}\right)$, which were planar with shorter $\mathrm{CO}$ bond lengths and smaller $\mathrm{HCH}$ angles. This effect is also seen in $\mathrm{HCO}^{-}$(Table VIII). In this species, the HOMO is a mixture of the carbon and oxygen lone pairs, with the less stable carbon lone pair more easily depopulated. Thus even the excitations of mostly $n_{\mathrm{O}\left(2 p_{x}\right)}$ character, similar to those in formaldehyde, also depopulate the adjacent $n_{\mathrm{C}\left(2 p x_{y}\right)} \mathrm{NBO}$. In fact, if enough density is removed from the valence region to Rydberg orbitals, the two adjacent $\mathrm{C}$ and $\mathrm{O} 2 p_{x y}$ lone pairs, each left with only $1.2-1.5 e$, are better described as a third $\mathrm{CO}$ bond of both $\sigma$ and $\pi$ character with occupancy of nearly 2.0 coupled with a less populated $\sigma^{*} / \pi^{*}$ orbital. This increased bonding causes the shorter bond length, and is accompanied by an increase in the $\mathrm{HCO}$ angles.

\section{EXCITED STATE DEPROTONATION ENERGIES}

For each acid-base pair, the energy difference between ground state optimized minima of the protonated acid $\mathrm{BH}^{+}$ and deprotonated base $\mathrm{B}$ is the ground state deprotonation energy, or electronic contribution to the proton affinity

$$
\Delta E_{0}\left(\mathrm{BH}^{+}\right)=-E_{0}\left(\mathrm{BH}^{+}\right)+E_{0}(\mathrm{~B}) .
$$

The base $\mathrm{B}$ may be an anion. This value differs from the experimental proton affinity by the vibrational, rotational, and translational contributions. These factors are generally found to decrease the calculated deprotonation energies by a few $\mathrm{kcal} / \mathrm{mol}$ due to partial cancellation between protonated and deprotonated species. ${ }^{19}$

For $\mathrm{CH}_{2} \mathrm{CH}_{2}$ we obtained a ground state deprotonation energy of $422.6 \mathrm{kcal} / \mathrm{mol}$, which is consistent with previous calculated values of $418-424 \mathrm{kcal} / \mathrm{mol}$ (Ref. 19) and higher than the experimental result of $406.0 \mathrm{kcal} / \mathrm{mol} .{ }^{53}$ The protonation energy of ground state $\mathrm{CH}_{2} \mathrm{NH}$ at the $\mathrm{HF} / 6-31$ $+\mathrm{G}(d, p)$ level, $222.90 \mathrm{kcal} / \mathrm{mol}$, is higher than the MP2/6-31+ $\mathrm{G}^{*}(d, p)$ value of $216.8 \mathrm{kcal} / \mathrm{mol}$ (Ref. 23) and other calculated values in the range of $200-208 \mathrm{kcal} / \mathrm{mol}$ at $298 \mathrm{~K}$ using various $a b$ initio methods. ${ }^{23,29}$ The experimental proton affinity ${ }^{54}$ of $\mathrm{CH}_{2} \mathrm{NH}$ is $204.1 \mathrm{kcal} / \mathrm{mol}$.

The deprotonation energy of $\mathrm{CH}_{2} \mathrm{OH}^{+}$at the HF/6-31 $+\mathrm{G}(d, p)$ level, $184.4 \mathrm{kcal} / \mathrm{mol}$, confirms the value at the same level of theory obtained by Del Bene, ${ }^{23}$ and is higher than the MP2/6-31+ $\mathrm{G}(d, p)$ value of $176.6 \mathrm{kcal} / \mathrm{mol}$, as well as the $\mathrm{G} 2$ value, ${ }^{28} 168.7 \mathrm{kcal} / \mathrm{mol}$. Calculations of the $\mathrm{CH}_{2} \mathrm{O}$ proton affinity are in the range of $167-179 \mathrm{kcal} / \mathrm{mol}$ for a variety of different approaches. ${ }^{20,21,23-27,55}$ These values are all within a few $\mathrm{kcal} / \mathrm{mol}$ of the experimental value, ${ }^{56} 171.7$ $\mathrm{kcal} / \mathrm{mol}$. Correlation decreases the deprotonation energy by $6-8 \mathrm{kcal} / \mathrm{mol}$, and the change in zero point and vibrational energy decreases the proton affinity by $6-10 \mathrm{kcal} / \mathrm{mol}$. Our deprotonation energies are close to those from previous calculations, and can be compared with those of the species in their excited states.

The excited state protonations which we consider are those which preserve the symmetry, character and, if possible, geometry of the state. Planar symmetry groups ${ }^{57}$ with some common symmetry elements can be mapped onto one another, as shown in Chart 1.

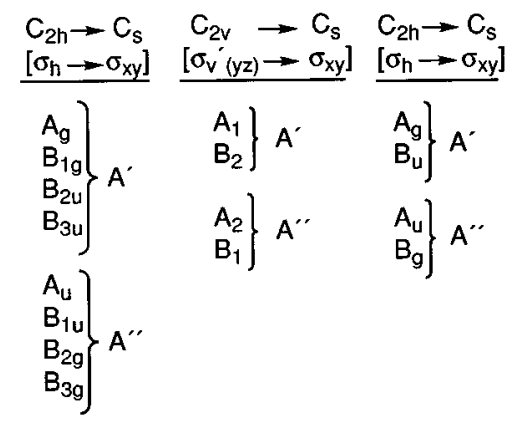

Excited state NBO analysis can be used as a helpful tool to "match" states of similar character (quantitatively similar depopulation and population of orbitals of the same type for both species) and compatible symmetry between the protonated and unprotonated species. The excited state adiabatic deprotonation energy may be written as

$$
\begin{aligned}
\Delta E_{k}^{A}\left(\mathrm{BH}^{+}\right)= & -E_{i}\left(\mathrm{BH}^{+}\right)+E_{j}(\mathrm{~B})=\Delta E_{0}\left(\mathrm{BH}^{+}\right) \\
& +\left[-E_{i}^{A}\left(\mathrm{BH}^{+}\right)+E_{j}^{A}(\mathrm{~B})\right],
\end{aligned}
$$

where $k$ is an index for excited state matches $i-j$ for which a deprotonation energy can be determined, $i$ is the excited state of protonated $\mathrm{BH}^{+}, j$ is the excited state of base $\mathrm{B}$. $E_{i}^{A}\left(\mathrm{BH}^{+}\right)$and $E_{j}^{A}(\mathrm{~B})$ are the adiabatic transition energies after the geometries of $\mathrm{BH}^{+}$and $\mathrm{B}$ have been optimized for states $i$ and $j$, respectively. A corresponding "vertical deprotonation energy" (VDE) can be calculated from the vertical transition energies, $E_{i}^{V}\left(\mathrm{BH}^{+}\right)$and $E_{j}^{V}(\mathrm{~B})$. This quantity shows the effect of electronic excitation independent of geometry relaxation.

Tables IX-XIII tabulate several vertical and adiabatic excited state deprotonation energies, ordered by the acid excited state, for the systems considered in this study, along with the ground state DE. Cations require much less energy to deprotonate; the ground state $\mathrm{DE}$ of $\mathrm{CH}_{2} \mathrm{NH}_{2}^{+}$is a little 
TABLE X. Excited state deprotonation energies (kcal/mol) for $\mathrm{CH}_{2} \mathrm{NH}_{2}^{+}$.

\begin{tabular}{|c|c|c|c|}
\hline Deprotonation reaction & Transition & $\begin{array}{c}\text { Vertical } \\
\text { deprotonation } \\
\text { energy }\end{array}$ & $\begin{array}{c}\text { Adiabatic } \\
\text { deprotonation } \\
\text { energy }\end{array}$ \\
\hline $\begin{array}{l}\text { Ground state } \\
\mathrm{CH}_{2} \mathrm{NH}_{2}^{+}(0) \rightarrow \mathrm{CH}_{2} \mathrm{NH}(0)+\mathrm{H}^{+}\end{array}$ & $\cdots$ & 222.9 & 222.9 \\
\hline Singlet states & & & \\
\hline$\underset{{ }^{{ }^{1} A_{1}}}{\mathrm{CH}_{2} \mathrm{NH}_{2}^{+}(2)} \rightarrow \underset{{ }^{1} A^{\prime}}{\mathrm{CH}_{2} \mathrm{NH}(3)}+\mathrm{H}^{+}$ & $\pi_{\mathrm{CN}} /\left[n_{\mathrm{N}}\right] \rightarrow \pi_{\mathrm{CN}}^{*}$ & 195.2 & 286.8 \\
\hline$\underset{{ }^{{ }^{1} B_{1}}}{\mathrm{CH}_{2} \mathrm{NH}_{2}^{+}(3)} \rightarrow \underset{{ }^{1} A^{\prime \prime}}{\mathrm{CH}_{2} \mathrm{NH}(10)}+\mathrm{H}^{+}$ & $\sigma_{\mathrm{CN}} / \sigma_{\mathrm{CH}} / \pi_{\mathrm{CN}} \rightarrow \pi_{\mathrm{CN}}^{*}$ & 193.6 & 202.3 \\
\hline 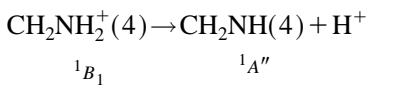 & $\pi_{\mathrm{CN}} \rightarrow r_{\mathrm{N}\left(3 s p_{x}\right)} / r_{\mathrm{C}\left(3 s p_{x}\right)} / \sigma_{\mathrm{NH}}^{*}$ & 154.0 & 308.4 \\
\hline $\begin{array}{c}\mathrm{CH}_{2} \mathrm{NH}_{2}^{+}(7) \rightarrow \mathrm{CH}_{2} \mathrm{NH}(7)+\mathrm{H}^{+} \\
{ }^{1} B_{1}\end{array}$ & $\pi_{\mathrm{CN}} \rightarrow r_{\mathrm{C}\left(3 s p_{y}\right)} /\left[r_{\mathrm{N}\left(3 s p_{y}\right)}\right] / \sigma_{\mathrm{CH}}^{*}$ & 129.2 & 316.1 \\
\hline$\underset{\mathrm{CH}_{2} \mathrm{NH}_{2}^{+}(9) \rightarrow \mathrm{CH}_{2} \mathrm{NH}(11)+\mathrm{H}^{+}}{{ }^{1} A_{1}}$ & $\pi_{\mathrm{CN}} \rightarrow \pi_{\mathrm{CN}}^{*} / r_{\mathrm{N}\left(3 p_{z}\right)} / r_{\mathrm{C}\left(3 p_{z}\right)}$ & 137.9 & 124.3 \\
\hline Triplet states & & & \\
\hline$\underset{\mathrm{CH}_{2} \mathrm{NH}_{2}^{+}(1)}{{ }^{3} A_{1}} \rightarrow \underset{{ }^{1} A^{\prime}}{\mathrm{CH}_{2} \mathrm{NH}}(1)+\mathrm{H}^{+}$ & $\pi_{\mathrm{CN}} \rightarrow \pi_{\mathrm{CN}}^{*}$ & 198.4 & 194.0 \\
\hline $\begin{array}{c}\mathrm{CH}_{2} \mathrm{NH}_{2}^{+}(3) \rightarrow \mathrm{CH}_{2} \mathrm{NH}(7)+\mathrm{H}^{+} \\
{ }^{3} B_{1}\end{array}$ & $\pi_{\mathrm{CN}} / \sigma_{\mathrm{CH}} / \sigma_{\mathrm{CN}} \rightarrow \pi_{\mathrm{CN}}^{*}$ & 199.2 & 193.5 \\
\hline $\begin{array}{c}\mathrm{CH}_{2} \mathrm{NH}_{2}^{+}(4) \rightarrow \mathrm{CH}_{2} \mathrm{NH}(4)+\mathrm{H}^{+} \\
{ }^{3} B_{1}\end{array}$ & $\pi_{\mathrm{CN}} \rightarrow \sigma_{\mathrm{NN}}^{*} / r_{\mathrm{N}(3 s)} / r_{\mathrm{C}(3 s)}$ & 160.9 & $\begin{array}{c}<0 \\
\text { (dissociative) }\end{array}$ \\
\hline$\underset{{ }^{3} B_{1}}{\mathrm{CH}_{2} \mathrm{NH}_{2}^{+}(9)} \rightarrow \underset{{ }^{2} A^{\prime \prime}}{\mathrm{CH}_{2} \mathrm{NH}(8)}+\mathrm{H}^{+}$ & $\pi_{\mathrm{CN}} \rightarrow r_{\mathrm{C}\left(3 s p_{y}\right)} / r_{\mathrm{N}\left(3 s p_{y}\right)} / \sigma_{\mathrm{CH}}^{*}$ & 134.5 & 194.0 \\
\hline
\end{tabular}

less than half of that of neutral ethylene. Each deprotonation reaction is labeled with the acid and base excited state and symmetry. Bracketed orbitals are those which make a minor contribution to the state of one species but not the other. For each reaction, the number of excited states that can be correlated between the protonated and deprotonated species indicates the similarity between the ground and excited state electronic structure of the two species. The $\mathrm{CH}_{2} \mathrm{CH}_{2} / \mathrm{CH}_{2} \mathrm{CH}^{-}$(Table IX), $\mathrm{CH}_{2} \mathrm{NH}_{2}^{+} / \mathrm{CH}_{2} \mathrm{NH}$ (Table X), and $\mathrm{CH}_{2} \mathrm{NH} / \mathrm{CH}_{2} \mathrm{~N}^{-}$(Table XI) systems show a fairly high degree of correlation. $\mathrm{CH}_{2} \mathrm{NH}$ and $\mathrm{CH}_{2} \mathrm{~N}^{-}$have the most excited states with similar character. In both species the HOMO is primarily an in-plane nitrogen lone pair. Though this lone pair has slightly different hybridization, $p_{x}$ for $\mathrm{CH}_{2} \mathrm{~N}^{-}$and $s p_{x} p_{y} \mathrm{CH}_{2} \mathrm{NH}$, excitations from this orbital are distinct in both species. In contrast to the $\mathrm{CH}_{2} \mathrm{NH} / \mathrm{CH}_{2} \mathrm{~N}^{-}$ system, the $\mathrm{CH}_{2} \mathrm{OH}^{+} / \mathrm{CH}_{2} \mathrm{O}$ system (Table XII), which nominally has the same bonding structure, shows much less correlation between the excited states. Only two singlet states and the relatively "pure" triplet $\pi \rightarrow \pi^{*}$ state have similar character. Few of the excited states of $\mathrm{CH}_{2} \mathrm{OH}^{+}$are stable, and the excitations in both species are much more delocalized over the entire molecule. The HOMO is no longer primarily a single lone pair on one center, but extends into the $\sigma_{\mathrm{CH}}$ and $\sigma_{\mathrm{OH}}$ bonds.

We focus our attention first on the vertical deprotonation energies in order to understand the effects of electronic excitation in isolation from nuclear arrangements. The excited state VDEs are almost all lower than those of the ground state. For most excited states, this can be attributed to the consistently larger "gap" between the ground and first excited state and the lower density of states for the protonated species than the deprotonated one. Tables I-VIII show that the first vertical excitation energy decreases over the series: protonated cation $>$ neutral species $>$ deprotonated anion. Figure 5 compares the triplet vertical excitation energies of $\mathrm{CH}_{2} \mathrm{NH}_{2}^{+}, \mathrm{CH}_{2} \mathrm{NH}$, and $\mathrm{CH}_{2} \mathrm{~N}^{-}$. The gap decreases over this series from 124.2 to 99.7 to $42.8 \mathrm{kcal} / \mathrm{mol}$, and the same trend in VEEs is seen for many excited states of similar character, such as the $\pi \rightarrow r_{\mathrm{C}\left(3 s p_{y}\right)} /\left[r_{\mathrm{N}\left(3 s p_{y}\right)} / \sigma_{\mathrm{CH}}^{*}\right]$ states, highlighted in Fig. 5.

One cause of the lowered DE in the excited state may be associated with the decreased charge on $\mathrm{X}_{2}$ due to the charge shift toward $C_{1}$ upon excitation of the base species. The lowered negative charge leads to less electrostatic attraction between the proton and anion, and less energy is gained by protonation of $\mathrm{N}$. However, due to the different number of hydrogens on the $\mathrm{X}_{2}$ atom, the charge of this atom alone is not always a reliable indicator.

For each acid/base deprotonation pair, the vertical transition energies of the base are consistently closer together 
TABLE XI. Excited state deprotonation energies (kcal/mol) for $\mathrm{CH}_{2} \mathrm{NH}$.

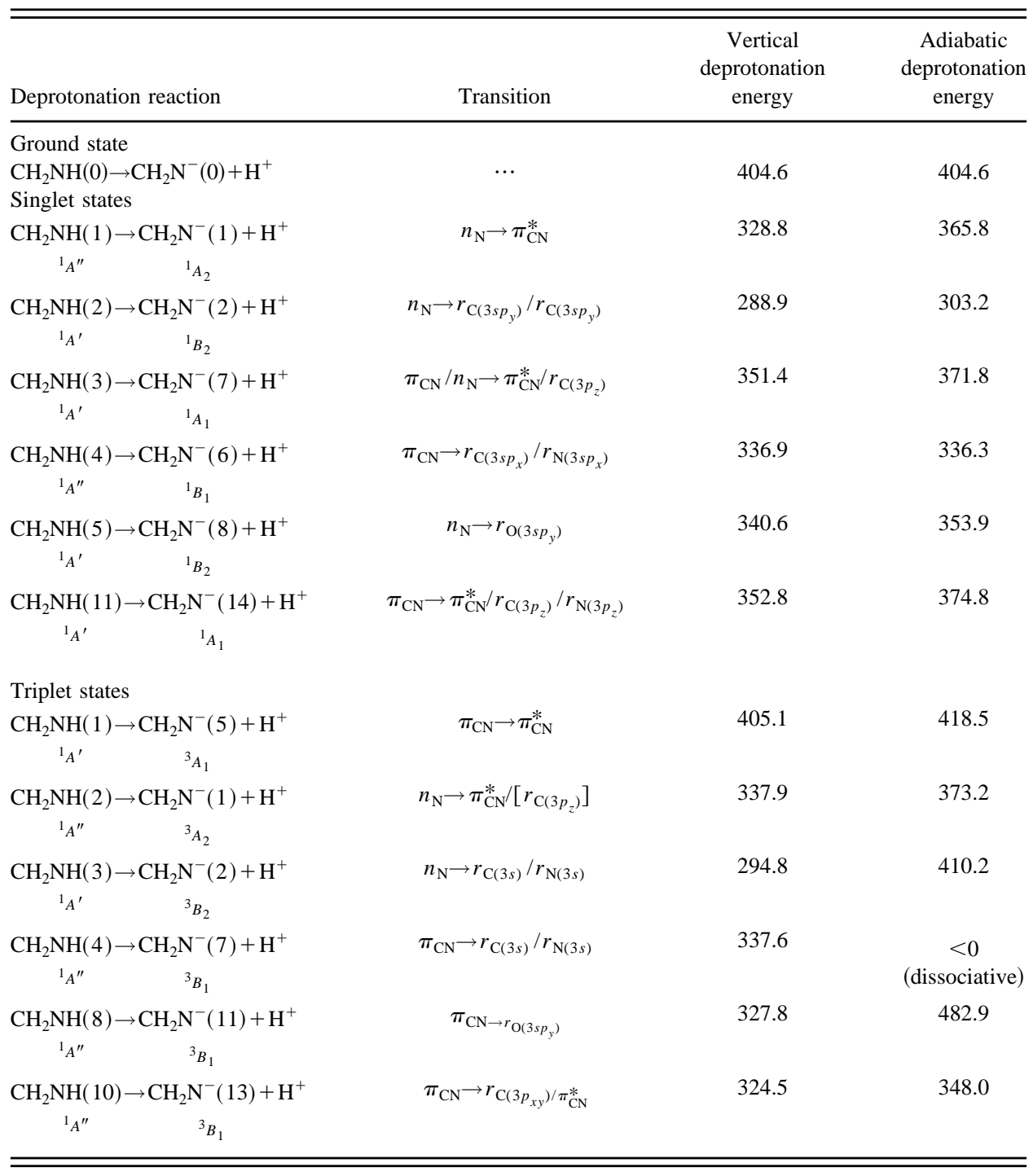

TABLE XII. Excited state deprotonation energies $(\mathrm{kcal} / \mathrm{mol})$ for $\mathrm{CH}_{2} \mathrm{OH}^{+}$.

\begin{tabular}{|c|c|c|c|}
\hline Deprotonation reaction & Transition & $\begin{array}{c}\text { Vertical } \\
\text { deprotonation } \\
\text { energy }\end{array}$ & $\begin{array}{c}\text { Adiabatic } \\
\text { deprotonation } \\
\text { energy }\end{array}$ \\
\hline \multicolumn{4}{|l|}{ Ground state } \\
\hline $\mathrm{CH}_{2} \mathrm{OH}^{+}(0) \rightarrow \mathrm{CH}_{2} \mathrm{O}(0)+\mathrm{H}^{+}$ & $\cdots$ & 184.4 & 184.4 \\
\hline \multicolumn{4}{|l|}{ Singlet states } \\
\hline $\mathrm{CH}_{2} \mathrm{OH}^{+}(3) \rightarrow \mathrm{CH}_{2} \mathrm{O}(6)+\mathrm{H}^{+}$ & $\pi_{\mathrm{CO}} /\left[n_{\mathrm{O}}\right] \rightarrow \pi_{\mathrm{CO}}^{*}$ & 159.0 & 239.2 \\
\hline${ }^{1} A_{1} \quad{ }^{1} A^{\prime}$ & & & \\
\hline $\mathrm{CH}_{2} \mathrm{OH}^{+}(8) \rightarrow \mathrm{CH}_{2} \mathrm{O}(8)+\mathrm{H}^{+}$ & $\sigma_{\mathrm{CH}} / n_{\mathrm{O}} \rightarrow \pi_{\mathrm{CO}}^{*} /\left[n_{\mathrm{C}\left(3 p_{x y}\right)} / r_{\mathrm{O}\left(3 s p_{x}\right)}\right]$ & 101.1 & 267.4 \\
\hline${ }^{1} A_{1} \quad{ }^{1} A^{\prime}$ & & & \\
\hline \multicolumn{4}{|l|}{ Triplet states } \\
\hline $\mathrm{CH}_{2} \mathrm{OH}^{+}(2) \rightarrow \mathrm{CH}_{2} \mathrm{O}(2)+\mathrm{H}^{+}$ & $\pi_{\mathrm{CO}} \rightarrow \pi_{\mathrm{CO}}^{*}$ & 143.9 & 158.6 \\
\hline${ }^{3} A_{1}$ & & & \\
\hline
\end{tabular}


TABLE XIII. Excited state deprotonation energies (kcal/mol) for formaldehyde.

\begin{tabular}{|c|c|c|c|}
\hline Deprotonation reaction & Transition & $\begin{array}{c}\text { Vertical } \\
\text { deprotonation } \\
\text { energy }\end{array}$ & $\begin{array}{c}\text { Adiabatic } \\
\text { deprotonation } \\
\text { energy }\end{array}$ \\
\hline \multicolumn{4}{|l|}{ Ground state } \\
\hline $\mathrm{CH}_{2} \mathrm{O}(0) \rightarrow \mathrm{HCO}^{-}(0)+\mathrm{H}^{+}$ & $\cdots$ & 408.2 & 408.2 \\
\hline \multicolumn{4}{|l|}{ Singlet states } \\
\hline$\underset{{ }^{1} \mathrm{CH}_{2} \mathrm{O}(4)}{{ }_{A_{1}}} \rightarrow \mathrm{HCO}^{-}(5)+\mathrm{H}^{+}$ & $n_{\mathrm{O}\left(2 p_{x}\right)} / \sigma_{\mathrm{CH}} /\left[n_{\mathrm{C}}\right] \rightarrow r_{\mathrm{C}\left(3 p_{x}\right)}$ & 291.5 & 327.6 \\
\hline $\begin{array}{c}\mathrm{CH}_{2} \mathrm{O}(9) \rightarrow \mathrm{HCO}^{-}(9)+\mathrm{H}^{+} \\
{ }^{1}{ }_{B_{1}} \\
{ }^{1} A^{\prime \prime}\end{array}$ & $\pi_{\mathrm{CO}} \rightarrow r_{\mathrm{C}(3 s)}$ & 344.7 & 341.6 \\
\hline \multicolumn{4}{|l|}{ Triplet states } \\
\hline 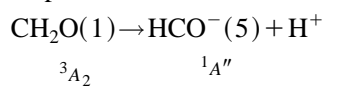 & $n_{\mathrm{O}\left(2 p_{x}\right)} /\left[n_{\mathrm{C}}\right] \rightarrow \pi_{\mathrm{CO}}^{*}$ & 414.7 & 413.2 \\
\hline $\begin{array}{c}\mathrm{CH}_{2} \mathrm{O}(2) \\
{ }^{3} A_{1}\end{array} \underset{\mathrm{HCO}^{-}(6)+\mathrm{H}^{+}}{{ }^{1} A^{\prime}}$ & $\pi_{\mathrm{CO}} \rightarrow \pi_{\mathrm{CO}}^{*}$ & 422.2 & 427.8 \\
\hline $\begin{array}{c}\mathrm{CH}_{2} \mathrm{O}(3) \\
{ }^{3} B_{2}\end{array} \underset{\mathrm{HCO}^{-}(2)+\mathrm{H}^{+}}{{ }^{1} A^{\prime}}$ & $n_{\mathrm{O}\left(2 p_{x}\right)} /\left[n_{\mathrm{C}}\right] \rightarrow r_{\mathrm{C}(3 s)} / \sigma_{\mathrm{CH}}^{*}$ & 275.0 & 374.2 \\
\hline 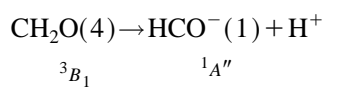 & $n_{\mathrm{O}\left(2 p_{x}\right)} /\left[n_{\mathrm{C}}\right] \rightarrow \pi_{\mathrm{CO}}^{*} / \sigma_{\mathrm{CO}}^{*}$ & 231.9 & 264.5 \\
\hline $\begin{array}{c}\mathrm{CH}_{2} \mathrm{O}(6) \\
{ }^{3} A_{1}\end{array}$ & $n_{\mathrm{O}\left(2 p_{x}\right)} / \sigma_{\mathrm{CH}} /\left[n_{\mathrm{C}}\right] \rightarrow r_{\mathrm{C}\left(3 p_{x}\right)} / r_{\mathrm{O}\left(3 p_{x}\right)}$ & 387.1 & 388.5 \\
\hline
\end{tabular}

than in the acid; however, many of those in the base are excitations out of the additional lone pair not common to both species. For example, the $\mathrm{CH}_{2} \mathrm{CH}^{-}$carbon lone pair is not present in ethylene. If one considers only those states common to both species, such as the ones connected by dot-

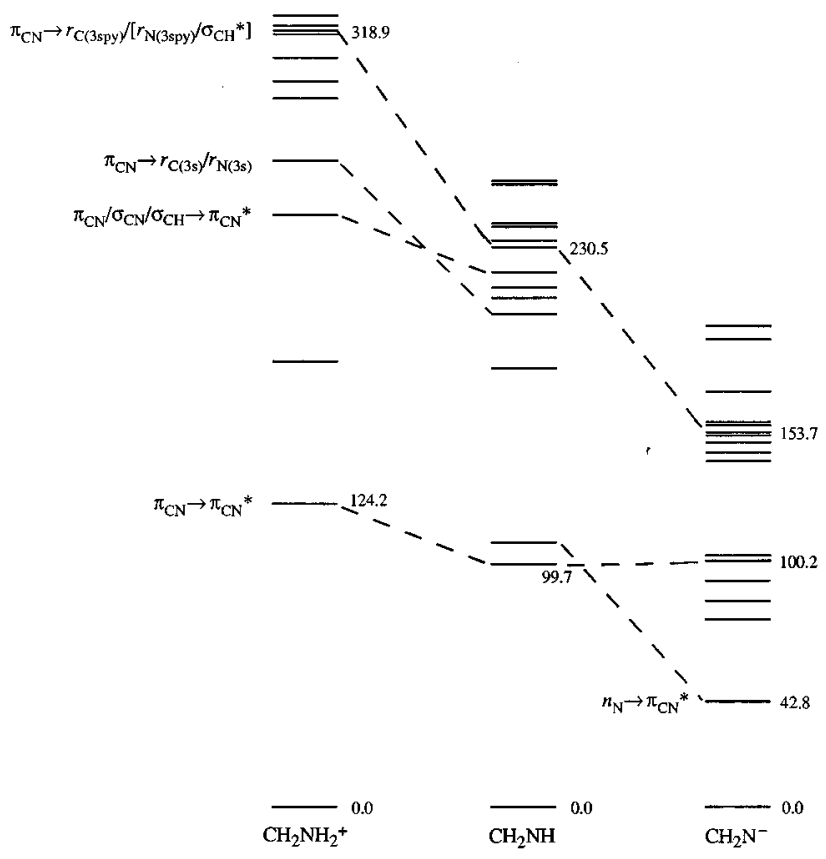

FIG. 5. Triplet state vertical transition energies (in $\mathrm{kcal} / \mathrm{mol}$ ) for the $\mathrm{CH}_{2} \mathrm{NH}_{n}$ series. Selected states for which a vertical deprotonation energy can be found are connected with dotted lines. ted lines in Fig. 5, the spacing of the excited states results in a general reduction of $\mathrm{VEE}$ as the system is progressively deprotonated. With one exception, the ordering of excited states of similar character is the same unless the states are close together in both species.

The one type of transition which does not follow the above trends is the $\pi \rightarrow \pi^{*}$ state. In some cases, this state undergoes little or no stabilization in the deprotonated species, as compared to the protonated one. An example is exhibited in Fig. 5, where the VEE of the $\pi \rightarrow \pi^{*}$ state in 21 $\mathrm{CH}_{2} \mathrm{~N}^{-}$is in fact slightly larger than in its parent $\mathrm{CH}_{2} \mathrm{NH}$. This behavior contrasts sharply with the lowering of most other states upon deprotonation. This is true of all the triplet and some singlet $\pi \rightarrow \pi^{*}$ states of the anions. The same phenomenon is noted in some singlet $\pi \rightarrow \pi^{*}$ states of neutral species as compared to their parent cation.

Investigation as to which MOs are involved in the $\pi \rightarrow \pi^{*}$ excitations which exhibit this phenomenon reveals that these excitations are indeed mostly of $\pi$ character, but also contain contributions from MOs with some $\sigma$ character. This contribution is also clearly seen in the occupancy changes of the $\sigma$-type NBOs, which are sometimes almost as great as those of the $\pi$ and $\pi^{*}$ orbitals. For example, in the case of the second triplet state listed in Table $\mathrm{X}$ $\left(\pi_{\mathrm{CN}} / \sigma_{\mathrm{CN}} / \sigma_{\mathrm{CH}} \rightarrow \pi_{\mathrm{CN}}^{*}\right)$, Fig. 5 shows that in the deprotonated species, $\mathrm{CH}_{2} \mathrm{NH}$, the state of this character, while lower in excitation energy than that of $\mathrm{CH}_{2} \mathrm{NH}_{2}^{+}$, is higher in order for $\mathrm{CH}_{2} \mathrm{NH}$ (state 7) than for $\mathrm{CH}_{2} \mathrm{NH}_{2}^{+}$(state 3). In addition to shifting density in the out-of-plane $\pi$ region from $\mathrm{X}_{2}$ to $\mathrm{C}_{1}$, density is depleted from the in-plane lone pair of $\mathrm{X}_{2}$ and one or more hydrogens to regions further from the 
molecule. Such shifts appear to destabilize the excited state more than pure $\pi \rightarrow \pi^{*}$ states in which this mixing does not occur. In the deprotonation reaction, this destabilization of the deprotonated species counteracts the general decrease in VEE accompanying the more negative net charge on the species, leading to a greater vertical deprotonation energy than the ground state $D E$, particularly for the $\mathrm{CH}_{2} \mathrm{CH}_{2}, \mathrm{CH}_{2} \mathrm{NH}$, and $\mathrm{CH}_{2} \mathrm{O}$ triplet $\pi \rightarrow \pi^{*}$ states.

Next we compare the effect of geometry relaxation on the process by comparing the vertical with the adiabatic DEs. There are four general types of geometry relaxation that occur after excitation of $\mathrm{H}_{2} \mathrm{C}=\mathrm{XH}_{n}$ species: changes in bond lengths (primarily $r_{\mathrm{CX}}$ ), changes in bond angles (primarily $\left.\theta_{\mathrm{CXH}}\right)$, "twisting" of the $\mathrm{XH}_{n}$ group relative to $\mathrm{CH}_{2}$, and pyramidal distortion of the $\mathrm{CH}_{2}$ group. Table I shows that the adiabatic excitation energies of the ethylene $1{ }^{1} B_{1 u}(V)$ and $1^{3} B_{1 u}(T)$ states have been lowered by 49.3 and $36.2 \mathrm{kcal} /$ mol, respectively, relative to the corresponding VEE values by twisting nearly $90^{\circ}$ [Fig. 2(a)]. The adiabatic transition energy of $2{ }^{3} B_{2 g}$ state [Fig. $2(\mathrm{c})$ ], is $55.1 \mathrm{kcal} / \mathrm{mol}$ lower due to an asymmetric pyramidal distortion of the $\mathrm{CH}_{2}$ groups. Nearly the same energy lowering, $46.4 \mathrm{kcal} / \mathrm{mol}$, occurs for the planar $2{ }^{1} B_{2 g}$ state, but in this case by a severe elongation of the CC bond by $0.31 \AA$. Twisting by $38.4^{\circ}$ lowers the energy of the $3{ }^{1} A^{\prime \prime}$ state of $\mathrm{CH}_{2} \mathrm{NH}$ (Table IV) by $25.8 \mathrm{kcal} /$ mol, while pyramidal distortion stabilizes several states by more than $40 \mathrm{kcal} / \mathrm{mol}$. Closure of the $\theta_{\mathrm{CNH}}$ angle from $112^{\circ}$ to $90^{\circ}$ with a slightly shorter $\mathrm{CN}$ bond stabilizes the $4{ }^{1} A^{\prime}$ state by $23.6 \mathrm{kcal} / \mathrm{mol}$, while opening it up to $144^{\circ}$ lowers the $4^{3} A^{\prime}$ state energy by $17.1 \mathrm{kcal} / \mathrm{mol}$. Bond length changes of less than $0.1 \AA$ and angle changes less than $10^{\circ}$ for other planar states lower the energy by only $\sim 5-15 \mathrm{kcal} / \mathrm{mol}$. Correlation of the amount of energy lowering with geometry changes in all eight species in this study yields the following stabilization hierarchy:

$\Delta r_{\mathrm{CX}} \approx \Delta \theta_{\mathrm{CXH}}$

$<$ pyramidal distortion $<$ twist $<$ twist + pyramidization .

Comparison of the major geometry changes of the protonated and deprotonated species upon relaxation explains the differences between the vertical and adiabatic excited state deprotonation energies. A greater geometric stabilization of the protonated species causes $E_{i}^{A}\left(\mathrm{BH}^{+}\right)$to be lower relative to $E_{j}^{A}(B)$, and hence the adiabatic deprotonation energy is greater than the VDE; the opposite is true if the deprotonated species is more stabilized. For the $n \rightarrow \pi^{*}$, $n \rightarrow r_{3 p_{z}}$ (out-of-plane Rydberg), all but one of the $\pi \rightarrow \pi^{*}$, and several of the $\pi \rightarrow r_{3 p_{z}}$ and $\pi \rightarrow r_{3 p_{y}}$ states, the optimized geometry of the protonated species shows nearly equal or greater twist or pyramidal distortion than does the deprotonated species. In several cases the increase of the deprotonation energy due to geometry relaxation is greater than is the lowering due to the change in the electron distribution (vertical properties), so the adiabatic deprotonation energy is even greater than that of the ground state. For the $\sigma \rightarrow \pi^{*}$ singlet and triplet states of ethylene, on the other hand, the deprotonated species $\left(\mathrm{CH}_{2} \mathrm{CH}^{-}\right)$is more distorted, so the
ADE is less than the VDE and is much smaller than the ground state deprotonation energy. The geometries of the acid and base are both planar for $\pi \rightarrow r_{3 p_{x}}, n \rightarrow r_{3 p_{x}}$, and $n \rightarrow r_{3 p_{y}}$ states, so the adiabatic deprotonation energy is within $\pm 20 \mathrm{kcal} / \mathrm{mol}$ of the VDE; the ADE is lower than the ground state deprotonation energy mainly due to the electronic effect.

\section{CONCLUSIONS}

Natural bond orbital analysis of CIS ground and excited state densities has been used to examine changes in bonding patterns upon excitation. This allows excited states of similar character in the acid and base to be correlated. The nature of the bonding NBOs that are depopulated and those antibonding or Rydberg orbitals that are populated can also be used to predict qualitative bond length and bond angle changes, including likely bond dissociations. In the case of planar molecules, the degree to which the excitation involves $\pi$ or $\pi^{*}$ MOs also determines the amount of nonplanar twisting or pyramidalization the system undergoes. NBO analysis shows that higher energy transitions are also more delocalized. Moreover, as the $\mathrm{C}-\mathrm{X}$ electronegativity difference becomes greater, the excitations become more delocalized, and show more involvement of the $\sigma_{\mathrm{CX}}, \sigma_{\mathrm{CH}}$, and $\sigma_{\mathrm{XH}}$ bonds and antibonds. This greater contribution of the $\sigma$ system to excitations leads to more frequent dissociation upon geometry relaxation.

The vertical transition energies of the deprotonated species tend to be lower and closer together than those of the protonated species. Therefore, most of the vertical deprotonation energies are less than the ground state DE. The calculations show evidence of a consistent charge shift away from the more electronegative atom upon excitation, which, independent of geometry relaxation, causes the species to be more easily deprotonated in the excited state than in the ground state in most cases. This shift becomes greater as the electronegativity difference between $\mathrm{C}$ and $\mathrm{X}$ increases. The exception is some $\pi \rightarrow \pi^{*}$ transitions, in which the VDE is higher than the ground state value because the excitation energy of the deprotonated species (anion or neutral) is higher than expected due to contributions from MOs with some $\sigma$-character which shift density away from the in-plane lone pair and hydrogens to regions further from the molecule.

Changes in geometry upon relaxation generally have a greater effect on the adiabatic deprotonation energies than shifts in the electron density, with pyramidal distortion and out-of-plane twisting more important than in-plane bond length and bond angle changes. The ADE may be greater or less than the VDE depending on whether the protonated or deprotonated species is more stabilized by geometry relaxation. Rydberg excited states, whose geometries generally remain planar, tend to decrease the deprotonation energy by removing electron density from the compact region about the heavy atom to which it is bonded. Unlike the adiabatic excited states in the $\mathrm{HC} \equiv \mathrm{CH} / \mathrm{HC} \equiv \mathrm{C}^{-}$and $\mathrm{HC} \equiv \mathrm{N} / \mathrm{C} \equiv \mathrm{N}^{-}$ systems, those of the $\mathrm{H}_{2} \mathrm{C}=\mathrm{XH}_{2}$ species tend to be ordered 
and spaced differently in the protonated and deprotonated species, and so excited state deprotonation energies can be up to $\sim 100 \mathrm{kcal} / \mathrm{mol}$ greater or less than the ground state deprotonation energy. The reordering is primarily caused by nonplanar distortion, which does not occur in these triply bonded systems.

These results are important in the context of proton transfer. To photocatalyze a rapid proton transfer, increasing the rate, one wishes to decrease the deprotonation energy or proton affinity of the proton donor, and increase that of the acceptor. In many cases this could be achieved experimentally by simultaneously exciting the donor to a low-lying Rydberg state and the acceptor into the $\pi^{*}$ orbital, providing the adiabatic geometry of the system allows a lower barrier. Since adiabatic geometry strongly influences the deprotonation energies, knowledge of the excited state deprotonation energies in a comprehensive set of useful prototype fragments of common biological molecules with analogous geometries can show how local geometry influences excited state proton transfer for the larger systems.

\section{ACKNOWLEDGMENT}

This work was supported by the National Science Foundation, Grant No. CHE-9123824.

${ }^{1}$ E. F. Caldin and V. Gold, Proton-Transfer Reactions (Halsted, New York, 1975); R. Stewart, The Proton: Applications to Organic Chemistry (Academic, Orlando, 1985).

${ }^{2}$ J. Rucker, Y. Cha, T. Jonsson, K. L. Grant, and J. P. Klinman, Biochemistry 31, 11489 (1992); A. J. Beveridge and G. C. Heywood, ibid. 32, 3325 (1993); I. A. Rose and D. J. Kuo, ibid. 31, 5887 (1992); D. N. Silverman and S. Lindskog, Acc. Chem. Res. 21, 30 (1988).

${ }^{3}$ M. Eigen, Angew. Chem. Int. Ed. Eng. 3, 1 (1964); L. Onsager, Science 166, 1359 (1969).

${ }^{4}$ L. G. Arnaut and S. J. Formosinho, J. Photochem. Photobiol. A 75, 1 (1993); S. J. Formosinho and L. G. Arnaut, ibid. 75, 21 (1993); D. A. Parthenopolous, D. McMorrow, and M. Kasha, J. Phys. Chem. 95, 2668 (1991); G. A. Brucker, T. C. Swinney, and D. F. Kelley, ibid. 95, 3190 (1991); J. Syage and J. Steadman, J. Chem. Phys. 95, 2497 (1991); P.-T. Chou, M. L. Martinez, and W. C. Cooper, J. Am. Chem. Soc. 114, 4943 (1992); T. Arthen-Engeland, T. Bultmann, N. P. Ernsting, M. A. Rodriguez, and W. Thiel, Chem. Phys. 163, 43 (1992).

${ }^{5}$ CIS: J. B. Foresman, M. Head-Gordon, J. A. Pople, and M. J. Frisch, J. Phys. Chem. 96, 135 (1992).

${ }^{6}$ MCSCF: B. O. Roos, in Methods in Computational Molecular Physics, edited by G. H. F. Diercksen and S. Wilson (Reidel, Dordrecht, 1983), p. 161; J. Olsen, D. L. Yeager, and P. Jorgenson, in Advances in Chemical Physics, edited by I. Prigogine and S. A. Rice (Wiley Interscience, New York, 1983), Vol. LIV, p. 1; H.-J. Werner and R. Shepard, in Advances in Chemical Physics, edited by K. P. Lawley (Wiley Interscience, New York, 1987), Vol. LXIX.

${ }^{7}$ MP2: C. Møller and M. S. Plesset, Phys. Rev. 46, 618 (1934); J. S. Binkley and J. A. Pople, Int. J. Quantum Chem. Symp. 99, 4899 (1977).

${ }^{8}$ S. Nagaoka, A. Itoh, K. Mukai, E. Hoshimoto, and N. Hirota, Chem. Phys. Lett. 192, 532 (1992); S. Roszak, U. Kaldor, D. A. Chapman, and J. J. Kaufmann, J. Phys. Chem. 96, 2123 (1992); Z. Latajka and S. Scheiner, ibid. 96, 9764 (1992).

${ }^{9}$ M. Kasha, Discuss. Faraday Soc. 9, 14 (1950).

${ }^{10}$ K. B. Wiberg, C. M. Hadad, J. B. Foresman, and W. A. Chupka, J. Phys. Chem. 96, 10756 (1992).

${ }^{11}$ L. Serrano-Andres, M. Merchán, I. Nebot-Gil, R. Lindh, and B. O. Roos, J. Chem. Phys. 98, 3151 (1993)

${ }^{12}$ E. R. Davidson and L. E. McMurchie, in Excited States, edited by E. C. Lim (Academic, New York, 1982), Vol. 5.

${ }^{13}$ J. Cioslowski, Int. J. Quantum Chem. 39, 681 (1991).
${ }^{14}$ C. M. Hadad, J. B. Foresman, and K. B. Wiberg, J. Phys. Chem. 97, 4293 (1993).

${ }^{15}$ M. Head-Gordon, R. J. Rico, M. Oumi, and T. J. Lee, Chem. Phys. Lett. 219, 21 (1994).

${ }^{16}$ Y. Dimitrova and S. D. Peyerimhoff, Chem. Phys. Lett. 227, 384 (1994).

${ }^{17}$ A. J. Merer and R. S. Mulliken, Chem. Rev. 69, 639 (1969), and references therein.

${ }^{18}$ M. B. Robin, Higher Excited States in Polyatomic Molecules (Academic, New York, 1985), Vol. III, pp. 253-260.

${ }^{19}$ J. Chandresekhar, J. G. Andrade, and P. v. R. Schleyer, J. Am. Chem. Soc. 103, 5609 (1981), and references therein.

${ }^{20}$ S. Olivella, F. Urpi, and J. Vilarrasa, J. Comput. Chem. 5, 230 (1984).

${ }^{21}$ M. J. S. Dewar and K. M. Dieter, J. Am. Chem. Soc. 108, 8075 (1986).

${ }^{22}$ G. P. Ford and J. D. Scribner, J. Comput. Chem. 4, 594 (1983).

${ }^{23}$ J. E. Del Bene, J. Comput. Chem. 6, 296 (1985).

${ }^{24}$ J. L. Ozment and A. M. Schmiedekamp, Int. J. Quantum Chem. 43, 783 (1992).

${ }^{25}$ P. Burk, K. Herodes, I. Koppel, and I. Koppel, Int. J. Quantum Chem. Symp. 27, 633 (1993).

${ }^{26}$ P. Speers and K. E. Laidig, J. Chem. Soc. Perkin Trans. II 4, 799 (1994).

${ }^{27}$ B. J. Smith and L. Radom, Chem. Phys. Lett. 231, 345 (1994).

${ }^{28}$ B. J. Smith and L. Radom, J. Am. Chem. Soc. 115, 4885 (1993).

${ }^{29}$ J. F. Gal, I. Leito, P. C. Maria, E. D. Raczyńska, R. W. Taft, and F. Anvia, J. Chim. Phys. 92, 22 (1995).

${ }^{30}$ V. Marudarajan and S. Scheiner, J. Phys. Chem. 95, 10280 (1991).

${ }^{31}$ V. Marudarajan and S. Scheiner, Chem. Phys. Lett. 186, 356 (1991).

${ }^{32}$ (a) J. P. Foster and F. Weinhold, J. Am. Chem. Soc. 102, 7211 (1980); (b) A. E. Reed and F. Weinhold, J. Chem. Phys. 78, 4066 (1983); (c) A. E. Reed, R. B. Weinstock, and F. Weinhold, ibid. 83, 735 (1985); (d) A. E. Reed and F. Weinhold, ibid. 83, 1736 (1985). Reviews: (e) A. E. Reed, L. A. Curtiss, and F. Weinhold, Chem. Rev. 88, 899 (1988); (f) F. Weinhold and J. E. Carpenter, in The Structure of Small Molecules and Ions, edited by R. Naaman and Z. Vager (Plenum, New York, 1988).

${ }^{33}$ NBO Program Version 4.0: E. D. Glendening, J. K. Badenhoop, A. E. Reed, J. E. Carpenter, and F. Weinhold, Theoretical Chemistry Institute, University of Wisconsin, Madison, WI, 1994. F. Weinhold, NBO 4.0 Program Manual (University of Wisconsin Theoretical Chemistry Institute, Madison, 1994). NBO Program Version 3.0: E. D. Glendening, A. E. Reed, J. E. Carpenter, and F. Weinhold, QCPE Bull. 10, 58 (1990); A. E. Reed and F. Weinhold, ibid. 5, 141 (1985).

${ }^{34}$ GAussian 92 Revision F.2: M. J. Frisch, G. W. Trucks, M. Head-Gordon, P. M. W. Gill, M. W. Wong, J. B. Foresman, B. G. Johnson, H. B. Schlegel, M. A. Robb, E. S. Repogle, R. Gomperts, J. L. Andres, K. Raghavachari, J. S. Binkley, C. Gonzalez, R. L. Martin, D. J. Fox, D. J. Defrees, J. Baker, J. J. P. Stewart, and J. A. Pople, Gaussian, Inc., Pittsburgh, PA, 1992.

${ }^{35}$ P. C. Hariharan and J. A. Pople, Theor. Chim. Acta 28, 213 (1973); J. D. Dill and J. A. Pople, J. Chem. Phys. 62, 2921 (1975); T. Clark, J. Chandrasekhar, G. W. Spitznagel, and P. v. R. Schleyer, J. Comput. Chem. 4, 294 (1983).

${ }^{36}$ J. Chandrasekhar, J. G. Andrade, and P. v. R. Schleyer, J. Am. Chem. Soc. 103, 5609 (1981); G. W. Spitznagel, T. Clark, J. Chandrasekhar, and P. v. R. Schleyer, J. Comput. Chem. 3, 363 (1982); D. J. DeFrees and A. D. McLean, ibid. 7, 321 (1986).

${ }^{37}$ Z. Latajka and S. Scheiner, Chem. Phys. Lett. 105, 435 (1984).

${ }^{38}$ Z. Latajka, S. Scheiner, and G. Chalasinski, Chem. Phys. Lett. 196, 384 (1992)

${ }^{39}$ J. M. L. Martin, J. P. François, and R. Gijbels, J. Comput. Chem. 10, 346 (1989).

${ }^{40}$ L. E. McMurchie and E. R. Davidson, J. Chem. Phys. 7, 2959 (1977).

${ }^{41}$ J. E. Del Bene, R. Ditchfield, and J. A. Pople, J. Chem. Phys. 55, 2236 (1971); R. Ditchfield, J. E. Del Bene, and J. A. Pople, J. Am. Chem. Soc. 94, 703 (1972).

${ }^{42}$ Pyridine and porphin: Ref. 5. Bicyclo[1.1.0]butane: V. A. Walters, C. M. Hadad, Y. Thiel, S. D. Colson, K. B. Wiberg, P. M. Johnson, and J. B. Foresman, J. Am. Chem. Soc. 113, 4782 (1991). 1,3-Butadiene: K. A. Wiberg, C. M. Hadad, G. B. Ellison, and J. B. Foresman, J. Phys. Chem. 97, 13586 (1993).

${ }^{43}$ I. Mayer, J. Mol. Struct. (Theochem) 255, 1 (1992).

${ }^{44}$ For a discussion of some problems associated with the use of nonorthogonal orbitals, see F. Weinhold and J. E. Carpenter, J. Mol. Struct. (Theochem) 165, 189 (1988). In particular, anomalous unphysical population values $(<0.00$ or $>2.00)$ violating the Pauli principle are found to 
increase sharply with the degree of orbital overlap [R. S. Mulliken and W. C. Ermler, Diatomic Molecules: Results of Ab Initio Calculations (Academic Press, New York, 1977), pp. 33-38]. Though natural population analysis [Ref. 32(c)] has been shown to give stable populations for ground state densities, orthogonalization of the distorted and highly overlapping excited state CIS AOs yields similar anomalies. Almost all CIS densities produced orbitals within an established occupancy limit of $-0.01<\rho$ $<2.01 e$; states yielding occupancies outside this limit were not considered.

${ }^{45}$ Natural bond orbitals have been shown to be highly transferable among similar molecules in the ground state [J. E. Carpenter and F. Weinhold, J. Am. Chem. Soc. 110, 368 (1988)], and for these transitions, NBOs describing relatively unaffected parts of the molecule are likewise transferable. For example, the overlap between the ground and excited state $\alpha_{\mathrm{CH}}$ bonds for the first excited triplet $\left(\pi \rightarrow \pi^{*}\right)$ state is 0.9997 , while that for the $\pi_{\mathrm{CC}}$ bond in the sixth singlet $\left(\sigma_{\mathrm{CH}} \rightarrow \pi^{*}\right)$ state is 0.9998 . Therefore, more compact NBO-based assignments are a direct indication of the similarity of the ground and excited state densities.

${ }^{46}$ R. J. Buenker and S. D. Peyerimhoff, Chem. Phys. Lett. 36, 415 (1975).

${ }^{47}$ Reference 18 , pp. $213-227$.

${ }^{48}$ R. S. Mulliken, Acc. Chem. Res. 9, 7 (1976); Chem. Phys. Lett. 46, 197 (1977).

${ }^{49}$ P. D. Foo and K. K. Innes, J. Chem. Phys. 60, 4582 (1974).

${ }^{50}$ All of the states which break the NH bonds populate one or both $\sigma_{\mathrm{NH}}^{*}$ orbitals or depopulate one or both $\sigma_{\mathrm{NH}}$ bonds by more than about $0.3-0.4$ $e$ relative to the ground state.

${ }^{51}$ For example, this can be seen in the case of the singlet $n_{\mathrm{X}_{2}} \rightarrow r_{\mathrm{C}_{1}\left(3 p x_{y}\right)}$ excitation. In $\mathrm{CH}_{2} \mathrm{CH}^{-}\left({ }^{1} A^{\prime}\right), 0.886 e$ is depleted from the $\mathrm{CH}_{2} \mathrm{CH}^{-}$ lone pair, and the Rydbergs on $\mathrm{C}_{1}$ and $\mathrm{C}_{2}$ increase occupancy by 0.509 and $0.304 e$, respectively, whereas in $\mathrm{CH}_{2} \mathrm{NH}\left(5^{1} A^{\prime}\right), 0.767 e$ depleted from the $\mathrm{N}$ lone pair is divided $0.641 / 0.087$ between the corresponding $\mathrm{C}$ and $\mathrm{N}$ Rydbergs. Thus, the charge on $\mathrm{C}_{1}$ becomes significantly more negative for this state in $\mathrm{CH}_{2} \mathrm{NH}$ than $\mathrm{CH}_{2} \mathrm{CH}^{-}\left(\Delta q_{\mathrm{C}_{1}}=-0.532\right.$ vs -0.389$)$.

${ }^{52}$ For example, the increase in charge shift toward $\mathrm{C}_{1}$ for the triplet $\pi \rightarrow \pi^{*}$ excitation $\left(\Delta q_{\mathrm{C}_{1}}=+0.376\right.$ for $\mathrm{CH}_{2} \mathrm{CH}^{-} 2^{3} A^{\prime \prime},-0.158$ for $\mathrm{CH}_{2} \mathrm{NH}^{3}{ }^{3} A^{\prime}$, and -0.738 in $\mathrm{CH}_{2} \mathrm{OH}^{+} 1^{3} A^{\prime}$ ) is consistent with the relative percentage composition of the ground state $\pi \mathrm{NBO}$ from the $\mathrm{C}_{1}$ vs $\mathrm{X}_{2} 2 p_{z}$ hybrids: 64:35 $\mathrm{C}_{1} / \mathrm{X}_{2}$ in $\mathrm{CH}_{2} \mathrm{CH}^{-}, 42: 58$ in $\mathrm{CH}_{2} \mathrm{NH}$, and 31:69 in $\mathrm{CH}_{2} \mathrm{OH}^{+}$; the coefficients are reversed for the $\pi^{*}$ orbital.

${ }^{53}$ S. G. Lias, J. E. Bartmess, J. L. Holmes, R. D. Levin, J. F. Liebman, and W. G. Mallard, J. Phys. Chem. Ref. Data 17, Suppl. N1, 673 (1988).

${ }^{54}$ In Ref. 53, p. 663.

${ }^{55}$ P. Kebarle, Annu. Rev. Phys. Chem. 28, 445 (1977).

${ }^{56}$ S. G. Lias, T. F. Liebman, and R. D. Levin, J. Chem. Phys. Ref. Data 13, 695 (1984).

${ }^{57}$ F. A. Cotton, Chemical Applications of Group Theory (WileyInterscience, New York, 1971). 